\title{
Pre-reproductive Parental Enriching Experiences Influence Progeny's Developmental Trajectories
}

\author{
Debora Cutuli,2*, Erica Berretta ${ }^{1,2+}$, Daniela Laricchiuta ${ }^{1,2}$, Paola Caporali ${ }^{1,2}$, \\ Francesca Gelfo ${ }^{2,3}$ and Laura Petrosini ${ }^{1,2}$ \\ ${ }^{1}$ Department of Psychology, Faculty of Medicine and Psychology, Sapienza University of Rome, Rome, Italy, ${ }^{2}$ Fondazione \\ Santa Lucia, Rome, Italy, ${ }^{3}$ Department of Human Sciences, Guglielmo Marconi University, Rome, Italy
}

While the positive effects of environmental enrichment (EE) applied after weaning, in adulthood, during aging, or even in the presence of brain damage have been widely described, the transgenerational effects of pre-reproductive EE have been less examined. And yet, this issue is remarkable given that parental environmental experience may imprint offspring's phenotype over generations through many epigenetic processes. Interactions between individual and environment take place lifelong even before conception. In fact, the environment pre-reproductively experienced by the mother and/or the father exerts a substantial impact on neural development and motor

OPEN ACCESS

Edited by: Anthony John Hannan,

Florey Institute of Neuroscience and Mental Health, Australia

Reviewed by:

Annabel Katherine Short, University of California, Irvine

United States

Irini Skaliora,

Biomedical Research Foundation of the Academy of Athens, Greece

*Correspondence: Debora Cutuli debora_cutuli@yahoo.it; debora.cutuli@uniroma1.it

${ }^{\dagger}$ Behavioral Neuroscience Ph.D.

Programme

Received: 08 May 2018 Accepted: 10 October 2018 Published: 12 November 2018

Citation:

Cutuli D, Berretta E, Laricchiuta D, Caporali P, Gelfo F and Petrosini L (2018) Pre-reproductive Parental Enriching Experiences Influence Progeny's Developmental Trajectories. Front. Behav. Neurosci. 12:254. doi: 10.3389/fnbeh.2018.00254 and cognitive performances of the offspring, even if not directly exposed to social, cognitive, physical and/or motor enrichment. Furthermore, pre-reproductive parental enrichment exerts a transgenerational impact on coping response to stress as well as on the social behavior of the offspring. Among the effects of pre-reproductive parental $E E$, a potentiation of the maternal care and a decrease in global methylation levels in the frontal cortex and hippocampus of the progeny have been described. Finally, pre-reproductive EE modifies different pathways of neuromodulation in the brain of the offspring (involving brain-derived neurotrophic factor, oxytocin and glucocorticoid receptors). The present review highlights the importance of pre-reproductive parental enrichment in altering the performances not only of animals directly experiencing it, but also of their progeny, thus opening the way to new hypotheses on the inheritance mechanisms of behavioral traits.

Keywords: environmental enrichment, maternal care, motor behavior, cognition, BDNF, oxytocin, stress response, rats

\section{INTRODUCTION}

Organisms adapt their physiology and behavior in response to environmental modifications. The influence of environmental experiences during lifespan is confined not only to the neurobehavioral profiles of the directly exposed individual, but it can also be evident in the next generations (Arai and Feig, 2011; Thayer and Kuzawa, 2011; Lim and Brunet, 2013). Growing evidence demonstrates that both ancestral and parental histories are able to affect the offspring depending on the valence of the environmental circumstances and the period of exposure to them, and that environmental influences may be passed on to the next generations through epigenetic (i.e., non-DNA sequence-based rather than mutational) modifications (Jirtle and Skinner, 2007; Caldji et al., 2011; Bohacek and Mansuy, 2015; 
Wang et al., 2017; Weaver et al., 2017). These studies shed new light on the Lamarckian theories of the inheritance of acquired traits that have been a matter of debate for over a century (Dröscher, 2015; Wang et al., 2017).

It is well-known that pre-natal/early post-natal as well as ancestral experience of environmental insults (e.g., undernutrition, stress) may result in modifications of the phenotype later in life (Shachar-Dadon et al., 2009; Franklin and Mansuy, 2010; Veenendaal et al., 2012; Xu et al., 2016; Ambeskovic et al., 2017). In the present review article we would address the effects of pre-reproductive enriching stimulations experienced by parents, such as the exposure to environmental enrichment (EE) or even exercise, on the behavioral and neurobiological phenotype of the offspring in rodents.

Environmental enrichment is an experimental paradigm apt to potentiate social, cognitive, and sensorimotor stimulations experienced by animals (Rosenzweig et al., 1964). By increasing environmental novelty and complexity, EE exerts potential therapeutic and neuroprotective effects as demonstrated by its efficacy in enhancing neural plasticity and delaying the progression and/or ameliorating the symptoms in the presence of brain injuries and diseases (Nithianantharajah and Hannan, 2006; Baroncelli et al., 2009; Nithianantharajah and Hannan, 2009; Petrosini et al., 2009; Cutuli et al., 2011; Simpson and Kelly, 2011; Sale et al., 2014; Mandolesi et al., 2017; Sampedro-Piquero and Begega, 2017; Gelfo et al., 2018). The broad beneficial effects of EE probably result from complex interactions among time window of exposure to EE, type of enrichment, and gender of enriched animals (Girbovan and Plamondon, 2013). On the other hand, scattered negative outcomes have also been reported, probably linked to the enhanced stress levels induced by the EE protocol (Schilling et al., 2004; Wood et al., 2011; Huzard et al., 2015; Mo et al., 2016).

Environmental enrichment as well as exercise may improve learning and memory, and enable neuroplasticity processes involving increased neurogenesis, possibly via neurotrophinmediated mechanisms (Kempermann et al., 1997; van Praag et al., 1999; Olson et al., 2006; Bechara and Kelly, 2013; Livingston-Thomas et al., 2016). Notably, EE and exercise effects are difficult to disentangle, because most enrichment paradigms incorporate exercise elements. Anyway, many studies demonstrated that the motor component (i.e., voluntary running-wheel exercise) is the major neurogenic and neurotrophic stimulus in $\mathrm{EE}$ protocols in comparison to the cognitive and social components (i.e., complex environments comprised of inanimate objects with or without social interactions, but without running-wheel) (Kobilo et al., 2011; Mustroph et al., 2012; Bechara and Kelly, 2013; Grégoire et al., 2014).

In the last years, attention has been paid to the transfer of proactive effects of enriching experiences from parents to the progeny, and the number of researches on this issue is significantly increased over time (Arai and Feig, 2011; Girbovan and Plamondon, 2013; Sale et al., 2014; Taouk and Schulkin, 2016; Sale, 2018). By using different rat and mouse strains, schedules and protocols of physical, social and/or cognitive enrichment, and various behavioral tests, these studies demonstrated that parental positive manipulations are able to alter the neurodevelopmental trajectories of the progeny, likely to prepare the fetus to cope with a specific environment.

The effects of parental enrichment across generations have been mainly investigated by exposing mothers to different kinds of environmental stimulations during gestation and/or lactation (Sale et al., 2014).

A rising literature demonstrates the role of maternal (Dell and Rose, 1987; Arai et al., 2009; Leshem and Schulkin, 2012; Caporali et al., 2014, 2015; Cutuli et al., 2015, 2017, 2018) or paternal (Mashoodh et al., 2012; Mychasiuk et al., 2012; Dezsi et al., 2016; Short et al., 2017; Yeshurun et al., 2017) enrichment "before conception" (i.e., during the prereproductive period) in modifying the neurobiological and behavioral profile of the offspring. Since early nurturing experiences may influence brain plasticity and alter epigenome (Meaney, 2010; Weaver et al., 2017), part of these studies have also evaluated if the effects of pre-reproductive parental enrichment on offspring's phenotype were mediated by modifications of maternal behavior (Mashoodh et al., 2012; Caporali et al., 2015; Cutuli et al., 2015, 2017, 2018; Short et al., 2017; Yeshurun et al., 2017).

To our knowledge, the literature on the effects of early EE by parents in humans is still scarce, and principally deals with fetuses' and newborns' stimulation. In fact, besides an accumulating evidence on the beneficial consequences of tactile (i.e., body massage) and auditory stimulation (i.e., exposure to maternal voice) on the neurobehavioral development of preterm infants (Guzzetta et al., 2009; Picciolini et al., 2014; Webb et al., 2015) or the effects of antenatal auditory stimulations (i.e., training with music and maternal talk to the fetus during pregnancy) on the reduction of autistic-like behaviors (Ruan et al., 2018), to date only one article has demonstrated the proactive influence of prereproductive enrichment represented by maternal educational achievement on buffering offspring's stress sensitivity (Swartz et al., 2018).

In the following paragraphs, we will take into account the impact of pre-reproductive parental exposure to enriching experiences on maternal behavior and on physical, motor, cognitive and emotional features of the offspring in rodents. Furthermore, based on our studies and the few others present in literature, we will focus on the changes in a key neurotrophic factor, such as the brain-derived neurotrophic factor (BDNF), and in the oxytocinergic system following pre-reproductive parental housing in highly stimulating environments. Whenever possible, comparisons with the effects of parental enrichment during gestational and/or lactation periods (i.e., post-reproductive enrichment) will be also considered. Namely, in Table 1 for each study we defined the period of enrichment exposure and if the enrichment was maternal and/or paternal, social (if the enriched cages contained more individuals than in the control groups), cognitive (if the objects, toys and other materials inside the enriched cages were systematically changed, rearranged or renewed), physical (if the enriched cages were bigger than the standard ones and 
TABLE 1 | Parental enrichment protocols.

Parental enrichment protocols

\begin{tabular}{|c|c|c|c|c|c|}
\hline \multicolumn{6}{|c|}{ A. Protocols of pre-reproductive parental enrichment } \\
\hline Authors & Year & Animals & Kind of enrichment & Enrichment period & $\begin{array}{l}\text { Kind of parental } \\
\text { enrichment }\end{array}$ \\
\hline Arai et al. (2009) & 2009 & Ras-grf k/o mice & $\begin{array}{l}\text { Moderate social, cognitive, physical } \\
\text { and motor enrichment (with } \\
\text { running-wheel) }\end{array}$ & Pre-reproductive period & $\begin{array}{l}\text { Maternal or } \\
\text { paternal }\end{array}$ \\
\hline Benito et al. (2018) & 2018 & C57Bl/6J mice & $\begin{array}{l}\text { Cognitive, physical and motor } \\
\text { enrichment (with running-wheels) }\end{array}$ & Pre-reproductive period & Paternal \\
\hline Caporali et al. (2014) & 2014 & Wistar rats & $\begin{array}{l}\text { Social, cognitive, physical and } \\
\text { motor enrichment (with } \\
\text { running-wheel) }\end{array}$ & Pre-reproductive period & Maternal \\
\hline Caporali et al. (2015) & 2015 & Wistar rats & $\begin{array}{l}\text { Social, cognitive, physical and } \\
\text { motor enrichment (with } \\
\text { running-wheel) }\end{array}$ & Pre-reproductive period & Maternal \\
\hline Champagne and Meaney (2007) & 2007 & Long-Evans rats & $\begin{array}{l}\text { Social, cognitive and physical } \\
\text { enrichment (without running-wheel) }\end{array}$ & Pre-reproductive period & Maternal \\
\hline Cutuli et al. (2015) & 2015 & Wistar rats & $\begin{array}{l}\text { Social, cognitive, physical and } \\
\text { motor enrichment (with } \\
\text { running-wheels) }\end{array}$ & Pre-reproductive period & Maternal \\
\hline Cutuli et al. (2017) & 2017 & Wistar rats & $\begin{array}{l}\text { Social, cognitive, physical and } \\
\text { motor enrichment (with } \\
\text { running-wheel) }\end{array}$ & Pre-reproductive period & Maternal \\
\hline Cutuli et al. (2018) & 2018 & Wistar rats & $\begin{array}{l}\text { Social, cognitive, physical and } \\
\text { motor enrichment (with } \\
\text { running-wheel) }\end{array}$ & Pre-reproductive period & Maternal \\
\hline Dezsi et al. (2016) & 2016 & $\begin{array}{l}\text { Genetic Absence } \\
\text { Epilepsy rats }\end{array}$ & $\begin{array}{l}\text { Cognitive, physical and motor } \\
\text { enrichment (with running-wheel) }\end{array}$ & Pre-reproductive period & Paternal \\
\hline Mashoodh et al. (2012) & 2012 & BALB/c mice & $\begin{array}{l}\text { Social, physical and motor } \\
\text { enrichment (with running-wheel) }\end{array}$ & Pre-reproductive period & Paternal \\
\hline Short et al. (2017) & 2017 & C57BI/6 mice & $\begin{array}{l}\text { Motor enrichment (by } \\
\text { running-wheel) }\end{array}$ & Pre-reproductive period & Paternal \\
\hline Yeshurun et al. (2017) & 2017 & C57BI/6J mice & $\begin{array}{l}\text { Cognitive and physical enrichment } \\
\text { (without running-wheel) }\end{array}$ & Pre-reproductive period & Paternal \\
\hline
\end{tabular}

B. Protocols of parental enrichment during pregnancy

\begin{tabular}{|c|c|c|c|c|c|}
\hline Authors & Year & Animals & Kind of enrichment & Enrichment period & $\begin{array}{l}\text { Kind of parental } \\
\text { enrichment }\end{array}$ \\
\hline Cymerblit-Sabba et al. (2013) & 2013 & Wistar rats & $\begin{array}{l}\text { Social, cognitive, physical and } \\
\text { motor enrichment with } \\
\text { running-wheel }\end{array}$ & Pregnancy & Maternal \\
\hline Gomes Da Silva et al. (2016) & 2016 & Wistar rats & $\begin{array}{l}\text { Motor enrichment (by motor driven } \\
\text { treadmill) }\end{array}$ & Pregnancy & Maternal \\
\hline Herring et al. (2012) & 2012 & TgCRND8 mice & $\begin{array}{l}\text { Motor enrichment (by } \\
\text { running-wheel) }\end{array}$ & Pregnancy & Maternal \\
\hline Kiyono et al. (1985) & 1985 & Fischer rats & $\begin{array}{l}\text { Social, cognitive and physical } \\
\text { enrichment (without running-wheel) }\end{array}$ & Pregnancy & Maternal \\
\hline Koo et al. (2003) & 2003 & Sprague-Dawley rats & $\begin{array}{l}\text { Social, cognitive, physical and } \\
\text { motor enrichment (with } \\
\text { running-wheel) }\end{array}$ & Pregnancy & Maternal \\
\hline Lee et al. (2006) & 2006 & Sprague-Dawley rats & Motor enrichment (by swimming) & Pregnancy & Maternal \\
\hline McKim and Thompson (1975) & 1975 & Sprague-Dawley rats & Physical and social enrichment & Pregnancy & Maternal \\
\hline Park et al. (2013) & 2013 & C57BI/6J mice & $\begin{array}{l}\text { Motor enrichment (by motor driven } \\
\text { treadmill) }\end{array}$ & Pregnancy & Maternal \\
\hline Parnpiansil et al. (2003) & 2003 & Sprague-Dawley rats & $\begin{array}{l}\text { Motor enrichment (by motor driven } \\
\text { treadmill) }\end{array}$ & Pregnancy & Maternal \\
\hline Rosenfeld and Weller (2012) & 2012 & WKY and Wistar rats & $\begin{array}{l}\text { Cognitive, physical and motor } \\
\text { enrichment (with running-wheel) }\end{array}$ & Pregnancy & Maternal \\
\hline
\end{tabular}


TABLE 1 | Continued

Parental enrichment protocols

C. Protocols of parental enrichment during pregnancy and lactation or only during lactation

\begin{tabular}{|c|c|c|c|c|c|}
\hline Authors & Year & Animals & Kind of enrichment & Enrichment period & $\begin{array}{l}\text { Kind of parental } \\
\text { enrichment }\end{array}$ \\
\hline Bick-Sander et al. (2006) & 2006 & C57Bl/6 mice & $\begin{array}{l}\text { Motor enrichment (by } \\
\text { running-wheel) }\end{array}$ & Pregnancy and lactation & Maternal \\
\hline Branchi et al. (2010) & 2010 & CD-1 mice & $\begin{array}{l}\text { Social and physical enrichment } \\
\text { (without running-wheel) }\end{array}$ & Pregnancy and lactation & Maternal \\
\hline Cancedda et al. (2004) & 2004 & $\begin{array}{l}\text { C57BI/6J mice, } 148 \text { CRE-LacZ } \\
\text { transgenic mice }\end{array}$ & $\begin{array}{l}\text { Social, cognitive and physical } \\
\text { enrichment (without } \\
\text { running-wheel) }\end{array}$ & Pregnancy and lactation & Maternal \\
\hline Durán-Carabali et al. (2018) & 2018 & Wistar rats & $\begin{array}{l}\text { Social, cognitive, physical and } \\
\text { motor enrichment (with } \\
\text { running-wheel) }\end{array}$ & Pregnancy and lactation & Maternal \\
\hline Heiderstadt et al. (2014) & 2014 & $\begin{array}{l}\text { C57BL/6J, DBA/2J and } \\
129 \times 1 / S v J \text { mice }\end{array}$ & $\begin{array}{l}\text { Social enrichment (without } \\
\text { running-wheel) }\end{array}$ & Lactation & Maternal \\
\hline Sale et al. (2004) & 2004 & C57BI/6J mice & $\begin{array}{l}\text { Social, cognitive, physical and } \\
\text { motor enrichment (with } \\
\text { running-wheel) }\end{array}$ & Pregnancy and lactation & Maternal \\
\hline Sparling et al. (2010) & 2010 & Long-Evans rats & $\begin{array}{l}\text { Physical and social enrichment } \\
\text { (without running-wheel) }\end{array}$ & Pregnancy and lactation & Maternal \\
\hline Welberg et al. (2006) & 2006 & Long-Evans rats & $\begin{array}{l}\text { Moderate physical enrichment } \\
\text { (without running-wheel) }\end{array}$ & Pregnancy and lactation & Maternal \\
\hline
\end{tabular}

D. Mixed protocols of parental enrichment

\begin{tabular}{|c|c|c|c|c|c|}
\hline Authors & Year & Animals & Kind of enrichment & Enrichment period & $\begin{array}{l}\text { Kind of parental } \\
\text { enrichment }\end{array}$ \\
\hline Bechard and Lewis (2016) & 2016 & $\begin{array}{l}\text { Peromyscus maniculatus (deer } \\
\text { mice) }\end{array}$ & $\begin{array}{l}\text { Social, cognitive, physical and } \\
\text { motor enrichment (with } \\
\text { running-wheel) }\end{array}$ & $\begin{array}{l}\text { Pre-reproductive and pregnancy } \\
\text { periods }\end{array}$ & $\begin{array}{l}\text { Maternal and } \\
\text { paternal }\end{array}$ \\
\hline Connors et al. (2015) & 2015 & Sprague-Dawley rats & $\begin{array}{l}\text { Physical and cognitive } \\
\text { enrichment (without } \\
\text { running-wheel) }\end{array}$ & $\begin{array}{l}\text { Pre-reproductive, pregnancy and } \\
\text { lactation periods }\end{array}$ & Maternal \\
\hline Curley et al. (2009) & 2009 & Balb/c mice & $\begin{array}{l}\text { Social and physical enrichment } \\
\text { (without running-wheel) }\end{array}$ & $\begin{array}{l}\text { Lactation (F0), pre-reproductive } \\
\text { period (F1) }\end{array}$ & Maternal \\
\hline Leshem and Schulkin (2012) & 2012 & Sprague-Dawley rats & $\begin{array}{l}\text { Social, cognitive, physical and } \\
\text { motor enrichment (with } \\
\text { running-wheels) }\end{array}$ & $\begin{array}{l}\text { Pre-reproductive period } \\
\text { (F0)/post-weaning (FO) }\end{array}$ & Maternal \\
\hline Maruoka et al. (2009) & 2009 & C57Bl/6J mice & $\begin{array}{l}\text { Cognitive, physical and motor } \\
\text { enrichment (with running-wheel) }\end{array}$ & $\begin{array}{l}\text { Pre-reproductive and pregnancy } \\
\text { periods }\end{array}$ & Maternal \\
\hline Mychasiuk et al. (2012) & 2012 & Long-Evans rats & $\begin{array}{l}\text { Social, cognitive and physical } \\
\text { enrichment (without } \\
\text { running-wheel) }\end{array}$ & $\begin{array}{l}\text { Pre-reproductive and pregnancy } \\
\text { periods (mothers), } \\
\text { pre-reproductive period (fathers) }\end{array}$ & $\begin{array}{l}\text { Maternal or } \\
\text { paternal }\end{array}$ \\
\hline Zuena et al. (2016) & 2016 & Wistar rats & $\begin{array}{l}\text { Social, cognitive, physical and } \\
\text { motor enrichment (with } \\
\text { running-wheel) }\end{array}$ & $\begin{array}{l}\text { Pre-reproductive and pregnancy } \\
\text { periods }\end{array}$ & Maternal \\
\hline
\end{tabular}

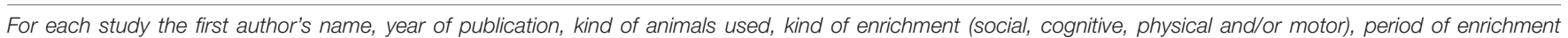

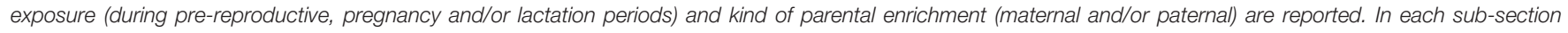
$(A-D)$, the studies are reported in alphabetical order.

contained objects, toys, igloos, tunnels, nesting materials, etc.) and/or motor (with one or more running-wheels). It is evident that in literature different EE paradigms has been used, with little understanding of how differences in individual EE variables (such as social, cognitive, physical and/or motor ones) might impact on specific downstream biological mechanisms. Thus, given the importance of the motor component in $\mathrm{EE}$ (as above discussed), exercise only paradigms (by running-wheel, motor driven treadmill or swimming) were also taken into account as forms of parental enriching experiences. In addition, we described the effects of communal nesting, a condition in which parental responsibilities are shared by multiple individuals in a nest, as a source of social extra-stimulation in pups' early life. 


\section{IMPACT OF DIFFERENTLY TIMED PARENTAL ENRICHMENT ON MATERNAL BEHAVIOR}

In mammals, the parental care ensures that the offspring effectively will survive until the reproductive age, thus transmitting the genetic information across generations. The maternal care in rodents is made up of a "constellation" of behaviors of preparation for the arrival of the newborns as well as their nurturing and protection (Kristal, 2009).

The literature especially reports maternal behavior alterations following various protocols of enrichment during gestational and/or lactation periods, while fewer studies are available on the effects of pre-reproductive parental enrichment.

\section{Effects of Maternal Enrichment During Pregnancy and/or Lactation}

Maternal exposure to EE during pregnancy and lactation increases maternal licking behavior, passive nursing and presence in the nest (Cancedda et al., 2004; Sale et al., 2004; DuránCarabali et al., 2018).

Lactating rat dams enriched only during gestation display more pup-directed behaviors than control-reared rats during the first and third lactation weeks, but decrease the nighttime frequency of presence in the nest and licking/grooming (LG) and arched-back nursing $(\mathrm{ABN})$ during the third lactation week (Rosenfeld and Weller, 2012).

Mother rats enriched before and during gestation show heightened LG behavior and reduced pup nursing (Zuena et al., 2016).

Maternal enrichment during pre-reproductive, gestational and lactation phases induces an increase in $\mathrm{ABN}$, but a reduction in the time spent in the nest and nursing (Connors et al., 2015).

There are also studies in which parental enrichment does not affect (Bechard and Lewis, 2016) or even worsen the maternal behavior (Welberg et al., 2006). In fact, the study by Bechard and Lewis (2016) used a biparental EE protocol in deer mice during pre-reproductive and gestational phases, and found no differences in maternal behavior of enriched mothers. The study by Welberg et al. (2006) used a moderate EE protocol in rats during pregnancy and lactation, and found no differences in pup licking, but reduced nursing episodes and presence in the nest.

Taken together these data, even somewhat contrasting, seem to account for a modulatory effect of environmental conditions on maternal care that reflects the different EE protocols used or the timing of enrichment exposure.

The more effective EE protocols for increasing maternal care are the ones providing at least social, cognitive and physical stimulations during pregnancy and lactation (Cancedda et al., 2004; Sale et al., 2004; Durán-Carabali et al., 2018). Moreover, when EE protocol involves the transition from the enriched gestational environment to the standard environment, the increase in some pup-directed behaviors may be a substitute to object exploration activity, given the decreased space and stimuli (Rosenfeld and Weller, 2012). A similar explanation can be valid also if females enriched in groups before and during gestation are individually housed in enriched cages near parturition (Zuena et al., 2016).

A variable conserved across dams housed in EE is the reduction in the time/frequency in contact with the nest. It may be attributed to the increased physical space of the enriched cage, when the EE protocol is applied at least during gestation and lactation (Welberg et al., 2006; Connors et al., 2015), given the enriched housing conditions reproduce the natural situation in which the dam is given more space to explore and thus more chance to leave the nest. On the contrary, the reduced maternal contact with the nest can be a sign of distress induced by the transition from the enriched gestational environment to the standard environment; this transition may have an aversive meaning leading to a premature withdrawal of $\mathrm{EE}$ dams from their pups (i.e., spending less time in the nest and performing less LG and lactation in the third post-natal week) (Rosenfeld and Weller, 2012). This behavioral pattern seems analogous to the early weaning manipulation, where the pups are separated from their mothers in the third post-natal week and become more anxious and stressful (Ito et al., 2006; Kikusui et al., 2006).

\section{Effects of Pre-reproductive Parental Enrichment}

Few researches have investigated the effects of pre-conceptional parental enrichment on maternal care to date. In particular, when rearing their own offspring under standard conditions, communally reared females are reported to perform enhanced levels of post-partum care in comparison to standard-reared females, and their offspring show increased frequency of nursing (Curley et al., 2009).

Post-weaning exposure to EE of low LG offspring enhances LG behavior and oxytocin receptor binding across generations (Champagne and Meaney, 2007).

Recently, we have demonstrated that pre-reproductive maternal EE induces maternal care modifications consisting of higher levels of licking, $\mathrm{ABN}$, and nest building activities, and a faster retrieving after maternal male intruder encounters (Cutuli et al., 2015, 2017, 2018). These effects of the pre-reproductive exposure of mothers to EE are accompanied by heightened levels of BDNF in the frontal cortex at pups' weaning (Caporali et al., 2015; Cutuli et al., 2015).

Paternal environmental experiences can modify offspring's phenotype even in the absence of paternal care. Unfortunately, only few studies addressed the role of mothers in the transmission of paternal effects by analyzing maternal care. Namely, standardreared female mice mated with male mice enriched during the pre-reproductive period show increased frequency of pup nursing and licking during the first post-partum week. Such behavioral modifications are associated with gene expression modifications (i.e., higher levels of BDNF mRNA and lower levels of MeCP2 mRNA) in the hypothalamus of dams (Mashoodh et al., 2012). The effects of paternal enrichment via maternal investment could be the consequence of inherited paternal epigenetic variations that lead to variations in the level of maternal care requested by the offspring. In fact, pups provide 
distal cues (e.g., sight, sound, tactile contact) for the mother, thus stimulating her contact with them. Interestingly, previous studies demonstrated that locomotion, ultrasonic vocalization and suckling ability are influenced by paternal genes (Curley et al., 2004; Plagge et al., 2004; Swaney, 2011). It can be speculated that the modulation of maternal behavior in standard-reared females mated with enriched males can be linked to a more demanding behavioral pattern of the pups.

Conversely, more recent studies did not find any effect of paternal enrichment on the maternal behavior (Short et al., 2017; Yeshurun et al., 2017).

Overall, the majority of the studies reported in this section seems to indicate that different pre-reproductive parental enriching experiences can potentiate the maternal care. Anyway, divergent data are emerged about the effects of pre-reproductive paternal enrichment on maternal behavior, possibly due to methodological reasons. In fact, in the study by Mashoodh et al. (2012) the fathers received increased social, physical and motor stimulations during their entire lifetime before breeding, and they were compared to male mice reared in isolated conditions. Differently, in the studies by Short and colleagues and Yeshurun and colleagues the fathers were exposed to only motor or only cognitive and physical enrichment and just in adulthood. Furthermore, in the study by Short et al. (2017) runner fathers and controls were both single-housed, while in the study by Yeshurun et al. (2017) enriched fathers and controls were both socially reared (four mice per cage).

It seems that the more is complete and long-lasting the set of stimulations provided to the fathers, more intense is the potentiating effects on the maternal behavior.

Furthermore, the use of socially isolated males (instead of standard-reared males) in the study by Mashoodh et al. (2012) could have contributed to shift the behavioral and neurobiological phenotypes of mates to extremes, thus influencing the maternal investment. Anyway, Mashoodh et al. (2012) showed that maternal investment is only partially dependent on the social and environmental experience of the mate (since environmentally induced anxiety levels of males did not fully predict the frequency of maternal nursing).

As evident, the implications of environmental factors on the complex relationship among paternal, maternal and offspring phenotypes still represent a crucial challenge in the study of the mechanisms driving paternal effects.

\section{IMPACT OF DIFFERENTLY TIMED PARENTAL ENRICHMENT ON THE OFFSPRING'S PHENOTYPE}

\section{Effects of Pre-reproductive vs. Post-reproductive Parental Enrichment on Physical Development and Motor Behavior}

A proper assessment of the transgenerational effects of parental experiences on offspring developmental trajectories can be achieved by examining the maturation of motor behaviors in rodents. This represents a useful tool to carefully assess early post-natal neurodevelopment, because the appearance of sensorimotor reflexes and motor skills typically follows a definite timing during the first 3 weeks after birth (De Souza et al., 2004).

Unfortunately, few studies paid specific attention to the transgenerational effects of EE on offspring's motor development and even fewer ones used the pre-reproductive EE paradigm, although it shows the possibility to distinguish pre- from postnatal effects of EE, thus allowing the study of Lamarckian inheritance.

To this aim, we performed a series of studies to examine the hypothesis that pre-reproductive maternal EE could affect progeny's phenotype, by rearing female Wistar rats in an enriched environment from weaning until mating (Caporali et al., 2014, 2015; Cutuli et al., 2015, 2017, 2018). To our knowledge, the study by Caporali and colleagues is the only available research analyzing the transgenerational effects of pre-reproductive maternal EE on offspring's development by using a battery of tests examining the acquisition of several developmental milestones in the physical and sensorimotor development (Caporali et al., 2014).

This pre-reproductive maternal EE did not affect litter characteristics, such as litter size and male/female ratio, thus suggesting that this experience does not influence the reproductive ability or the pregnancy of female rats (Caporali et al., 2014; Cutuli et al., 2015, 2017). Interestingly, similar results have been reported following differently timed maternal EE protocols (Welberg et al., 2006; Bechard and Lewis, 2016; Zuena et al., 2016) as well as following pre-reproductive paternal enrichment (Mashoodh et al., 2012; Mychasiuk et al., 2012; Yeshurun et al., 2017).

We found that pre-reproductive maternal enrichment affects offspring's body weight only at birth, with enriched mothers' offspring weight less than controls (Cutuli et al., 2015, 2017), similarly to their own enriched mother. As suggested by Sparling et al. (2010), this finding is consistent with researches demonstrating that enriched females are leaner (Pham et al., 1999; Larsson et al., 2002; Olsson and Dahlborn, 2002; Moncek et al., 2004; Brillaud et al., 2005) and maintain their weight stable over time (Brillaud et al., 2005). However, even opposing results have been collected. In fact, when female rats are exposed to a social colony designed to provide enhanced physical and social stimulations, during pregnancy and lactation, they give birth to heavier offspring (Sparling et al., 2010). Given higher weight of the offspring at birth is retained a good predictor of developmental success (Byrd and Weitzman, 1994; Prathanee et al., 2009), the authors conclude that heavier colony pups would have got a physiological advantage compared to their control mates (Sparling et al., 2010). No differences in the body weight have been reported in adulthood, even following different schedules of maternal enrichment (Welberg et al., 2006; Maruoka et al., 2009; Zuena et al., 2016). The pre-reproductive paternal exposure to enriched social, cognitive and physical stimulations (regardless running-wheel) does not affect body weight at birth, but it does predict male offspring's weight at adulthood, resulting in an increased weight gain (Mashoodh et al., 2012) that persists until the second generation (Yeshurun et al., 2017). 
Overall, it seems that parental environmental experiences affect the body weight of the progeny. Namely, enriched mothers influence progeny's body weight only at birth, in a way ambiguously linked to different EE conditions, whereas enriched fathers influence offspring's body weight at adulthood. Thus, it is possible to speculate that both maternal and paternal influences are driven by pre-natal metabolic programming, even if we cannot exclude that a specific maternal behavior in the preweaning phase may account for the particular fitness outcome observed in the progeny of enriched fathers (Mashoodh et al., 2012).

The pre-reproductive maternal EE alters the motor development of the progeny, leading to an earlier acquisition of those abilities that require complex sequencing and coordination of the motor output (Caporali et al., 2014). Furthermore, maternal enrichment does not influence the postural development or affect the appearance of dynamic sensorimotor reflexes (negative geotaxis, cliff avoidance, and vestibular drop). Interestingly, similar results on negative geotaxis acquisition were obtained by Mychasiuk et al. (2012) evaluating the effects of pre-reproductive paternal enrichment. Nevertheless, the same authors found that the offspring of females enriched prior to and during pregnancy exhibit a reduction in time required to show negative geotaxis, but no EE effects were recorded on the day of the appearance. Thus, one possibility is that maternal EE, only if gestational, affects the time required to show negative geotaxis as days go by. A similar effect on negative geotaxis performance has been reported also by combining pre- and post-natal EE: the maternal exposure to social, cognitive, physical and motor enrichment occurring during pregnancy and lactation is able to promote a better reflex performance (i.e., reduced latency) counteracting the neurobehavioral delay induced by neonatal hypoxia ischemia, a common neurological complication occurring in preterm infants (Durán-Carabali et al., 2018). Given some researches demonstrated that postnatal EE exposure positively influences negative geotaxis development (Kiss et al., 2013; Schuch et al., 2016), it is possible that direct exposure to EE of fetus first and pup later leads to a better performance, without affecting the day of the appearance.

The open field test (OF) is one of the most frequently used method to assay locomotor behavior in rodents and is sensitive to EE-induced exploratory modifications. The analysis of the effects of pre-reproductive parental enrichment on explorative activity of the progeny provided conflicting results. In fact, increased OF activity levels have been reported in young male offspring of pre-reproductively enriched fathers (Mychasiuk et al., 2012), while adult male offspring of pre-reproductively enriched mothers exhibit activity levels similar to controls (Cutuli et al., 2015). Opposite findings were also obtained with differently timed maternal EE protocols. Mychasiuk et al. (2012) found increased OF activity levels in young male and female offspring of mothers enriched prior to and during pregnancy. Even crossfostered pups of female rats exposed to EE during pregnancy show increased locomotion in the OF (McKim and Thompson, 1975). Anyway, reduced OF activity levels have been reported in young and adult female offspring in other experimental conditions (Maruoka et al., 2009; Rosenfeld and Weller, 2012). The conflicting findings now described, along with the wellknown influence exerted by several factors on OF activity levels [for a review see (Simpson and Kelly, 2011)], make this issue worthy of further investigation.

In conclusion, the exposure of rodents to EE during the prereproductive phase significantly shapes the neurodevelopment of progeny, by accelerating the acquisition of motor abilities (Caporali et al., 2014). No significant effects have been described on litter features (Mashoodh et al., 2012; Mychasiuk et al., 2012; Caporali et al., 2014; Cutuli et al., 2015, 2017; Yeshurun et al., 2017), while contrasting findings, probably linked to different EE protocols and experimental parameters, have been reported on body weight and OF activity levels.

\section{Effects of Pre-reproductive vs. Post-reproductive Parental Enrichment on Cognition and Anxiety Effects of Maternal Enrichment During Pregnancy
and/or Lactation}

In rodents the effects of parental enrichment on the offspring's cognitive performances and anxiety levels have been primarily investigated by exposing mothers to complex stimulations during pregnancy and/or lactation, and by using different kinds of behavioral tasks and biochemical correlates. Namely, the exposure of mothers to different kinds of EE paradigms during pregnancy facilitates learning and memory abilities (Kiyono et al., 1985; Koo et al., 2003), and increases synaptic plasticity and hippocampal neurogenesis in the progeny (Koo et al., 2003). In addition, the maternal EE exposure during pregnancy reduces attentional performance (Cymerblit-Sabba et al., 2013), and controversially affects anxiety levels of the offspring (Maruoka et al., 2009; Rosenfeld and Weller, 2012; Cymerblit-Sabba et al., 2013).

Maternal exposure to EE during pregnancy and lactation improves spatial memory performances in the Morris water maze, reduces anxiety, and prevents the hippocampal tissue loss after neonatal hypoxia ischemia (Sparling et al., 2010; DuránCarabali et al., 2018).

Also the exposure of mothers to exercise only paradigms during pregnancy (Parnpiansil et al., 2003; Lee et al., 2006; Herring et al., 2012) or during pregnancy and lactation (Bick-Sander et al., 2006) is able to improve learning and memory abilities, protect from neurodegeneration, improve brain plasticity and enhance hippocampal neurogenesis in the offspring.

The maternal exposure to EE before and during gestation affects offspring's development trajectories by modifying cognitive and emotional outcomes in a sex-specific manner with improved learning ability only in females and increased anxiety mainly in males (Connors et al., 2015; Zuena et al., 2016).

Lastly, data resulting from post-natal social enrichment obtained by communal nesting are variable and still 
difficult to reconcile across studies. For example, a study by Heiderstadt et al. (2014) does not find any difference induced by communal nesting in learning or anxiety tests in the adult offspring. Conversely, in a study by Curley et al. (2009) communal nesting reduces anxiety levels and modifies oxytocin and vasopressin receptor densities in the adult offspring. These results were obtained by using different mice strains [i.e., C57BL/6J, DBA/2J and $129 \times 1 /$ SvJ mice (Heiderstadt et al., 2014) vs. Balb/c mice (Curley et al., 2009)], and suggest the importance of considering strain-specific effects in interpreting the longterm developmental effects of early social enrichment. In fact, communal nesting may be particularly effective and beneficial when using the $\mathrm{Balb} / \mathrm{c}$ strain, since these mice display a more stress vulnerable phenotype [e.g., elevated stress responses and behavioral inhibition accompanied by reduced hippocampal glucocorticoid receptor expression and increased corticosterone (CORT) levels] (Brinks et al., 2007; Brodkin, 2007).

\section{Effects of Pre-reproductive Parental Enrichment}

The few studies available on the effects of pre-conceptional parental enrichment on the cognition and emotional response of the offspring generally indicate an amelioration of memory performances and a reduced anxiety. In fact, Arai et al. (2009) found rescued contextual fear conditioning memory and enhanced long-term potentiation not only in mice directly exposed to 2-weeks of EE when juveniles, but also in their offspring that never experienced EE. Benefits of EE across generations are associated with a signaling cascade in the CA1 hippocampal region and pass on through the mother (Arai et al., 2009). In rats, intergenerational effects of females' exposure to EE from weaning to reproductive age include improvements in cognitive performances as well as increased hippocampal BDNF levels in their male offspring, without changes in neurogenesis or reelin levels (Cutuli et al., 2015). A similar protocol of prereproductive maternal enrichment does not influence avoidance learning, and induces sex-dependent effects in female offspring by reducing anxiety and improving habituation to acoustic startle (Leshem and Schulkin, 2012).

Low LG female offspring housed under enriched conditions display increased LG behavior and higher levels of exploration in comparison to standard housed low LG females (Champagne and Meaney, 2007). These variations are also passed to the progeny.

In addition, communal nesting is able to increase maternal care and reduce anxiety across generations (Curley et al., 2009).

As for pre-reproductive paternal enrichment experiences, the existing studies mainly addressed the emotional responses of the offspring. Namely, a very recent study by Benito et al. (2018) demonstrated that pre-conceptional exposure of adult male mice to EE enhances LTP and induces a subtle memory improvement in the next generation, and that this phenotype is mediated by changes in the RNA composition in the sperm of the enriched fathers, especially through the upregulation of microRNA 212 and 132 .

In a genetic rat model of absence epilepsy, early enrichment of fathers from weaning to breeding induces anti-epileptogenic and anxiolytic effects that were heritable across generations (Dezsi et al., 2016). These findings are in line with the anxiolytic effects of pre-reproductive paternal exercise. In fact, the male offspring of runner fathers show reduced anxiety levels and more robust fear extinction memory associated with alterations in the levels of small non-coding RNAs in sperm (Short et al., 2017). Conversely, it has been recently demonstrated that when the motor enrichment component is lacking, no differences in anxiety are found following pre-reproductive paternal enrichment (Yeshurun et al., 2017).

In conclusion, it seems that pre-reproductive paternal housing conditions which include an overt motor enrichment by runningwheel presence are able to induce anxiolytic effects.

With regard to indirect effects of parental enriching experiences on the pups' behavioral trajectories through the modulation of maternal care, the few data currently available are still little explicative, since maternal behavior has not been systematically investigated. However, it can be noted that when an enhancement in maternal care is evident following prereproductive maternal enriching experiences, the offspring's phenotype can be characterized by enhanced maternal care and/or reduced anxiety (Champagne and Meaney, 2007; Curley et al., 2009) or by improved cognitive performances (Cutuli et al., 2015).

As for paternal enrichment, it seems that a clear relationship between maternal care and offspring's behavior is still not well-definite. In fact, studies reporting behavioral modifications in the offspring of not-enriched females mated with males enriched during the pre-reproductive period fail to report maternal care modifications, thus suggesting that the progeny could be differently influenced by the pre-reproductive paternal experiences according to the different environmental manipulations used (motor enrichment vs. cognitive and physical enrichment), regardless maternal investment (Short et al., 2017; Yeshurun et al., 2017). Interestingly, enriching fathers preconceptionally significantly reduced global methylation levels in the frontal cortex and hippocampus of the developing offspring (Mychasiuk et al., 2012). And, other studies found modifications of RNA expression in the sperm (Short et al., 2017; Benito et al., 2018). As sperm development in rodents occurs continuously, as in humans, it can be speculated that pre-reproductive paternal enrichment experiences are able to alter gene expression in the sperm of sires, thus providing a means for the transmission of epigenetic change to the progeny.

\section{Effects of Pre-reproductive vs. Post-reproductive Parental Enrichment on Social Behavior}

Social interactions are essential for survival and proper neural and behavioral development. After weaning, playful interactions with peers allow the acquisition of social and cognitive competence. Social play behavior is a highly rewarding activity in humans and animals, as it can instill a sense of well-being and pleasure, motivates approach behaviors toward a specific social stimulus, and finally elicits associative learning in order to attribute salience to socially related cues (Trezza et al., 2011; 
Vanderschuren et al., 2016). Interestingly, some studies indicate that EE may influence the social behavior in rodents. Namely, the exposure of pups to EE in the post-weaning period (MorleyFletcher et al., 2003; Leshem and Schulkin, 2012) increases social interaction at adulthood and adolescence, respectively. Also, maternal exposure to EE before and during gestation enhances social behavior in the adolescent offspring with contrasting results depending on sex [i.e., higher social contact duration in the female offspring (Connors et al., 2015), and higher social play behavior in the male offspring (Zuena et al., 2016)].

As for pre-reproductive maternal EE, two studies demonstrated that it reduces social interaction in males, but not in females either in adult and adolescent offspring (Leshem and Schulkin, 2012; Cutuli et al., 2018). Similar effects are found in the adult offspring of pre-reproductively stressed females (Leshem and Schulkin, 2012), and are in line with the generally increased vulnerability of male rats to developmental disruption of social behavior by gestational ethanol (Mooney and Varlinskaya, 2011). The reduction in social play, and in particular in play solicitation (i.e., Pouncing) found in the enriched dams' male adolescent offspring by Cutuli et al. (2018), is probably linked to a less rewarding value of play initiation (Vanderschuren et al., 1997) or to not clarified neurohormonal modifications.

Nevertheless, pre-reproductive maternal EE does not induce any difference in sociability as assessed in the three-chamber sociability test (Cutuli et al., 2015), in which social interaction is prevented and the animal has to choose between a compartment containing a juvenile conspecific or an empty compartment.

\section{Effects of Pre-reproductive vs. Post-reproductive Parental Enrichment on Stress Response}

Environmental enrichment has been considered as prevention/intervention strategy to counteract the negative consequences of stress (McCreary and Metz, 2016).

The ability to cope with environmental challenges is essential to be successfully adapted. To face acute and chronic stressors, the sympathetic system and the hypothalamic-pituitary-adrenal (HPA) axis guarantee the recruitment of necessary resources, the inhibition of non-necessary ones, and fine feedback mechanisms for homeostasis. Glucocorticoids (GCs) secreted from adrenal glands act peripherally and in the brain through their binding with mineralcorticoid (MR) and glucocorticoid (GR) receptors. GR are recruited when GCs levels are elevated, as in a stressful condition, and are involved in negative feedback on HPA axis (De Kloet et al., 2005; Herman et al., 2016; Mifsud and Reul, 2018).

Genetic background (Kundakovic et al., 2013; Andolina et al., 2015; Di Segni et al., 2016) and parental environment (Meaney, 2001) shape individual differences in the ability to cope with stress and in the susceptibility of its negative consequences (Belsky and Pluess, 2009; Karatsoreos and McEwen, 2011; Daskalakis et al., 2013; Boersma and Tamashiro, 2015).

Parents have a pivotal role in programming the offspring's HPA axis functioning as widely demonstrated in rodents (Meaney, 2001; Enthoven et al., 2010), and more controversially in non-human primates (Sanchez, 2006) and humans (Tollenaar et al., 2011).

Parental stress has been associated with offspring's greater risk of psychopathology (Glover, 2011), higher glucocorticoid sensitivity (Lehrner et al., 2014), immunological alterations (Laviola et al., 2004), enhanced neuronal activity (Bielas et al., 2014) and altered DNA methylation (Mulligan et al., 2012; Essex et al., 2013). Recently, epigenetic inheritance is assigned a captivating role in the intergenerational outcomes (Franklin et al., 2010), stressing the standing of "epigenetics prior to the birth" (Lo and Zhou, 2014).

Compelling evidence from animal models highlights the effectiveness of the EE in preventing, rescuing or normalizing the negative outcomes of stress (McCreary and Metz, 2016). In rodents post-weaning EE normalizes basal immune parameters altered by pre-natal stress (Laviola et al., 2004), reverses the negative effects of pre-natal stress on HPA axis reactivity and play behavior (Morley-Fletcher et al., 2003), attenuates CORT levels after restraint stress (Sztainberg et al., 2010) and exerts enduring effects on CORT daily pattern and levels in the response to a novel environment (Peña et al., 2009). Similar findings were also recently found in zebrafish (Danio rerio) (Marcon et al., 2018).

In rats long-lasting moderate maternal EE during pregnancy, and together with pups during lactation until weaning, modifies the response of the female offspring to a chronic stress (Welberg et al., 2006). Whereas chronically stressed females show increased basal CORT and reduced adrenocorticotropic (ACTH) levels in response to stress, no such effects neither on basal CORT nor in ACTH acute release are found in chronically stressed offspring exposed to early EE.

Moreover, the positive effects of $\mathrm{EE}$ on stress response are reported in different strains of mice. Branchi et al. (2010) research with outbred CD-1, Swiss-derived strain (ICR) of mice clearly demonstrates that social enrichment by communal nesting that provides maternal caregiving from three different mothers and higher peer interaction is able to modify the offspring's behaviors and neuroendocrine response to stress, by reducing anhedonia and decreasing CORT levels in response to social stress, enhancing time spent in immobility at the forced swim test (FST) and finally dampening the response to 3-weeks fluoxetine treatment. Also, in Balb/c mice, an inbred strain known for its high anxiety-like behavior, social enrichment by communal nesting reduces offspring's stress response when exposed to a novel environment (Curley et al., 2009).

Remarkably, the enhancement of stimulations provided by EE produces changes that propagate across generations influencing descendant's future responses to chronic and acute stress (Taouk and Schulkin, 2016) and promoting resilience even at the germline level (Gapp et al., 2016). The authors (Gapp et al., 2016) evidenced the role of both negative and positive environmental manipulations (i.e., maternal stress and unpredictable maternal separation, and exposure to EE, respectively) in influencing potential epigenome trajectories across generations. In particular, they proved that the detrimental effects of early stress on male offspring coping behaviors (F1) are related to a significant increase in GR expression in hippocampus and showed the propagation of the stress-related negative outcomes up until the 
subsequent generation (F2). The intergenerational transmission of phenotype is associated with a decreased methylation in exons 1-7 of the GR gene both in the F2 offspring and in the F1 sperm. In addition, the authors disclosed that $\mathrm{EE}$ is effective in preventing the transgenerational effects of stress on F2. The GR gene hypomethylation found in the sperm of early-stressed males was in fact rescued by EE. A very recent study also emphasizes the role of specific sperm microRNAs in mediating the effects of paternal pre-conceptional EE (Benito et al., 2018).

Pre-reproductive EE can modify the effect of social isolation, in rats. The offspring born to standardly reared mothers and subjected to social isolation from weaning to adolescence show greater GR expression in amygdala compared to notisolated controls, whereas socially isolated offspring born to pre-reproductively enriched mothers do not show any difference in GR expression when compared to the respective notisolated controls. Furthermore, a blunted amygdaloid c-Fos immunoreactivity in response to the FST is evident in the offspring of pre-reproductively enriched mothers in comparison to the offspring of standardly reared dams (Cutuli et al., 2017).

Recently, the transgenerational effects of pre-conceptional paternal $\mathrm{EE}$ on despair behaviors and neuroendocrine phenotypes of the adult offspring in mice has been investigated (Yeshurun et al., 2017). EE effects skip the F1 generation and are sex-specific. While pre-conceptional paternal EE has no effect on despair behaviors of F1 male and female offspring neither on basal ACTH or CORT levels in response to FST, modifies F2 females' behavioral despair and CORT response in the FST. The transgenerational effect of paternal EE is not mediated by paternally induced changes in maternal care since no differences in maternal behaviors between EE and standardly reared male paired mothers.

Leshem and Schulkin (2012) showed that pre-reproductive maternal enrichment ameliorates the anxiogenic effect induced by pre-reproductive stress on offspring. The above-mentioned study also demonstrates stress-like effects of EE.

Environmental enrichment and stress indeed activate similar circuitries and involve HPA axis activity and changes in GR expression and functionality (Larsson et al., 2002; Moncek et al., 2004).

Several theoretical models have been proposed to account for the beneficial or contrasting effects of $\mathrm{EE}$ on stress response. Crofton et al. (2015) had addressed the issue in the inoculation stress hypothesis framework: the enhanced and complex stimulation provided by EE would represent a continuous mild form of stress that inoculates animals to subsequent challenges, as a controlled exposure to an harmless vaccine would protect against future encounter of the disease. Besides, the transition from eustress to detrimental stress follows the non-linear inverted U-shaped dose-response curve for which optimal levels of functionality are obtained at the moderate CORT expression while very high (e.g., overstimulation) or very low (e.g., understimulation) levels can exert similar deleterious effects on the individual (Sapolsky, 2015).

Considering conflicting transgenerational EE effects, the degree of fitness between the environmental challenges experienced by the offspring and the environmental demands for which parents and grandparents have equipped future generations to cope with has to be keep in mind (Marshall and Uller, 2007; Daskalakis et al., 2012; Nederhof and Schmidt, 2012; Prizak et al., 2014).

Finally, transgenerational inheritance could interact with ingrained individual differences to determine the phenotypic scenarios "for better or for worse" (Belsky et al., 2009).

\section{IMPACT OF DIFFERENTLY TIMED PARENTAL ENRICHMENT ON BDNF}

Neurotrophins constitute a protein family whose components show analogous structure and exert an essential action on the development and function of the neurons. They regulate cell proliferation and differentiation, growth and readjustment of axons and dendrites, and plastic changes involved in synaptic function (Park and Poo, 2013). In particular, BDNF is retained a key-player in the translation of the experience in neural structure and function modifications and a trigger and mediator of synaptic plasticity (Cowansage et al., 2010; Bekinschtein et al., 2011; Aarse et al., 2016). The direct effects of EE on brain BDNF levels have been demonstrated in a large number of animal studies (Angelucci et al., 2009; Gelfo et al., 2011; Mosaferi et al., 2015; Novkovic et al., 2015).

Recently, it has been evaluated if the known EE effects on BDNF-mediated brain plasticity could be transgenerationally transmitted. Namely, some research has been specifically devoted to study the effects of the pre-reproductive exposure of mothers to EE on the offspring's BDNF brain levels (Caporali et al., 2014; Caporali et al., 2015; Cutuli et al., 2015). Interestingly, while the pre-reproductive maternal EE does not modify pups' brain BDNF protein levels at birth, changes are evident at weaning and in adulthood (Caporali et al., 2014; Cutuli et al., 2015). It may be hypothesized that eventual latent genetic and epigenetic effects of pre-reproductive maternal $\mathrm{EE}$ on BDNF signaling need the interaction with the mother and the external ambient (that occurs only after the birth) to be manifest in term of BDNF protein differences. In fact, stable effects of pre-reproductive maternal $\mathrm{EE}$ are evident in pups' brain BDNF levels from weaning onward, and are different in association with the different experiences the pups are exposed to. When BDNF brain expression is evaluated at weaning in pups exposed to repeated motor challenging and exercise, BDNF increases in enriched mother's offspring at cerebellar and striatal levels, demonstrating an enhanced plasticity expression in the areas involved in motor performance (Caporali et al., 2014). On the other hand, when BDNF expression is evaluated in pups exposed only to maternal care and cage interaction with brotherhood (and not to behavioral testing), BDNF increases at weaning and in adulthood only in hippocampus, the brain area involved in memory formation, and thus solicited by every experience (Cutuli et al., 2015). The exposure after birth to the care of an enriched mother that shows in turn increased brain BDNF level (Caporali et al., 2015; Cutuli et al., 2015) is fundamental for any BDNF change. In fact, the overt increase in hippocampal BDNF levels is not anymore found 
when the pups are born to an enriched mother but raised until weaning by a standard-reared mother (Caporali et al., 2015).

In line with our results, in a different model of maternal EE, pups born to mothers enriched during pregnancy and lactation do not show variations in cortical or hippocampal BDNF levels few days after birth (Durán-Carabali et al., 2018). In addition, it has been demonstrated that even a specific component of pre-reproductive EE, such as exercise, is able to change offspring's BDNF brain levels. Parnpiansil et al. (2003) reported that rat pups born to mother exposed to motor enrichment during pregnancy show BDNF mRNA hippocampal expression enhanced at birth, unchanged during lactation and reduced after weaning. More recently, it has been showed that motor enrichment during pregnancy induces enhanced BDNF protein expression in the hippocampus of pups when adult (Gomes Da Silva et al., 2016). Similarly, Park et al. (2013) showed that mice pups born to mother exposed to motor enrichment during pregnancy display enhanced hippocampal BDNF expression after weaning. Furthermore, maternal motor enrichment during pregnancy induces increased BDNF mRNA hippocampal expression in rat pups after weaning (Lee et al., 2006).

On the whole, the available evidence supports the influence of the maternal enrichment during pre-reproductive and post-reproductive periods on the pup brain BDNF-mediated neuroplasticity, regardless of the specific characteristics of the enrichment paradigm.

\section{IMPACT OF DIFFERENTLY TIMED PARENTAL ENRICHMENT ON THE OXYTOCINERGIC SYSTEM}

The neuropeptide oxytocin is widely implicated in the social behavior of mammalian by modulating maternal care, infant behavior, social bonding, agonistic behavior and social recognition (Lim and Young, 2006; Bosch, 2013; Crespi, 2016). It is synthesized in the magnocellular neurons of the hypothalamic paraventricular nucleus (PVN) and supraoptic nucleus (SON), and is transported along their axons to the posterior pituitary and released from there into the blood stream to act on target organs in the periphery (Veenema, 2009). It is also released in the forebrain and hindbrain regions (Veenema, 2009). Oxytocin receptors are expressed in many brain regions, including cortical, limbic, hypothalamic and brain stem areas (Lee et al., 2009; Veenema, 2009).

Unfortunately, there is a scant literature on the effects of the enrichment on oxytocinergic system either in the dams and offspring. With regards to differently enriched dams, it seems that a potentiation of the oxytocinergic system is linked to increased maternal care. For example, communally reared dams exhibit elevated levels of post-partum care and of oxytocin receptor density in the lateral septum across generations (Curley et al., 2009). Similarly, post-weaning enrichment enhances LG behavior and oxytocin receptor binding of low LG offspring in the PVN, medial preoptic area and bed nucleus of the stria terminalis in comparison to low LG offspring housed in standard conditions (Champagne and Meaney, 2007).

A recent study has demonstrated the impact of prereproductive maternal $\mathrm{EE}$ on the hypothalamic oxytocinergic neurons on mothers and pups (Cutuli et al., 2018). Indeed, enriched dams show an increased number of oxytocinergic neurons either in PVN and SON associated with increased crouching levels and faster pups' retrieval. As for the adolescent offspring, while no differences have been found in the female pups, the male pups of pre-reproductively enriched dams exhibited higher levels of oxytocinergic neurons in SON and reduced play behavior. Interestingly, the anti-aggressive properties of the oxytocin are well-documented in humans and rodents (Todeschin et al., 2009; Crespi, 2016; Hathaway et al., 2016), and SON is the hypothalamic nucleus selectively activated after the display of offense (Kollack-Walker et al., 1997). Thus, being the social play a sort of preparation for the adult aggressive behavior (Aldis, 1975), the reduction in play behavior observed in male pups of enriched dams may be attributed to reduced aggressive tendencies linked to increased oxytocin levels in SON.

Due to the consistent interactions between oxytocinergic and dopaminergic systems in the establishment and maintenance of mother-pup relationship (Crespi, 2016), the differently enriched dams could be more attracted by pups because of a more rewarding effect of nurturing behaviors. And in turn, their pups could be more soliciting. To our knowledge, only one study has described an increased motivation of male pups born to pre-reproductively enriched females to contact the odor of their mothers (Cutuli et al., 2015). Furthermore, the increased maternal care found in enriched dams may potentiate the oxytocinergic system of their pups through epigenetic modifications, as demonstrated in previous studies (Champagne, 2008).

In conclusion, many questions remain to be answered and further studies are expected to clarify the role of oxytocinergic system in mediating the impact of parental enrichment in the subsequent generations.

\section{DISCUSSION}

An increasing body of evidence from animal studies shows the outcomes of maternal enrichment exposure during, before and/or after the gestation on development, behavior and physiological functioning of the progeny (Arai and Feig, 2011; Taouk and Schulkin, 2016).

In the present review article, we focused on the effects of pre-reproductive exposure of parents to highly stimulating environments on maternal behavior and offspring's phenotype.

A problem encountered in screening the current (still limited) literature is the diversity of paradigms as for timing of exposure and enhanced stimulations used. Nevertheless, a common pattern emerges in findings. As for dams, the pre-reproductive enrichment tendentially results in increased maternal care, brain BDNF and oxytocinergic levels. As for offspring, the pre-reproductive parental enrichment appears to accelerate the acquisition of complex motor 
abilities, potentiate cognitive performances and coping skills, reduce anxiety, increase brain BDNF levels and modulate social behavior and oxytocinergic system in a sex-dependent manner.

\section{CONCLUSION}

On the whole, the available evidence supports an influence of pre-reproductive parental enrichment on mother's and pups' brain and behavior. However, given the diversity in the different parental enrichment paradigms, systematic analyses on the selective exposure to EE (and its social, cognitive, physical and motor components) in the different pre-reproductive and postreproductive periods are required to provide exhaustive evidence on brain and behavior changes induced in pups by parental experiences.

It is interesting to speculate on the mechanisms of inheritance involved in the transmission of the environmental influence from parents to the offspring. The inheritance across generations may involve epigenetic modifications in the germline or can be passed to the offspring through

\section{REFERENCES}

Aarse, J., Herlitze, S., and Manahan-Vaughan, D. (2016). The requirement of BDNF for hippocampal synaptic plasticity is experience-dependent. Hippocampus 26, 739-751. doi: 10.1002/hipo.22555

Aldis, O. (1975). Play Fighting. New York, NY: Academic press Inc, doi: 10.1016/ B978-0-12-049450-7.50018-X

Ambeskovic, M., Roseboom, T. J., and Metz, G. A. S. (2017). Transgenerational effects of early environmental insults on aging and disease incidence. Neurosci. Biobehav. Rev. doi: 10.1016/j.neubiorev.2017.08.002 [Epub ahead of print].

Andolina, D., Puglisi-Allegra, S., and Ventura, R. (2015). Strain-dependent differences in corticolimbic processing of aversive or rewarding stimuli. Front. Syst. Neurosci. 8:207. doi: 10.3389/fnsys.2014.00207

Angelucci, F., De Bartolo, P., Gelfo, F., Foti, F., Cutuli, D., Bossu, P., et al. (2009). Increased concentrations of nerve growth factor and brain-derived neurotrophic factor in the rat cerebellum after exposure to environmental enrichment. Cerebellum 8, 499-506. doi: 10.1007/s12311-009-0129-1

Arai, J. A., and Feig, L. A. (2011). Long-lasting and transgenerational effects of an environmental enrichment on memory formation. Brain Res. Bull. 85, 30-35. doi: 10.1016/j.brainresbull.2010.11.003

Arai, J. A., Li, S., Hartley, D. M., and Feig, L. A. (2009). Transgenerational rescue of a genetic defect in long-term potentiation and memory formation by juvenile enrichment. J. Neurosci. 29, 1496-1502. doi: 10.1523/JNEUROSCI.505708.2009

Baroncelli, L., Braschi, C., Spolidoro, M., Begenisic, T., Sale, A., and Maffei, L. (2009). Nurturing brain plasticity: impact of environmental enrichment. Cell Death Differ. 17, 1092-1103. doi: 10.1038/cdd.2009.193

Bechara, R. G., and Kelly, Á. M. (2013). Exercise improves object recognition memory and induces BDNF expression and cell proliferation in cognitively enriched rats. Behav. Brain. Res. 245, 96-100. doi: 10.1016/j.bbr.2013. 02.018

Bechard, A. R., and Lewis, M. H. (2016). Transgenerational effects of environmental enrichment on repetitive motor behavior development. Behav. Brain Res. 307, 145-149. doi: 10.1016/j.bbr.2016.04.005

Bekinschtein, P., Oomen, C. A., Saksida, L. M., and Bussey, T. J. (2011). Effects of environmental enrichment and voluntary exercise on neurogenesis, learning and memory, and pattern separation: BDNF as a critical variable? Semin. Cell Dev. Biol. 22, 536-542. doi: 10.1016/j.semcdb.2011.07.002 maternal care during early post-natal life (Champagne and Curley, 2009; Bohacek and Mansuy, 2015; Kundakovic and Champagne, 2015). Non-genetic marks fluctuate throughout lifetime and carry important information about previous experiences and faced environments, and their outcomes on the organism (Campos et al., 2014). For these reasons, exposure to enriching experiences may be regarded as therapeutic intervention to support healthy aging (Ambeskovic et al., 2017) or reverse stress detrimental effects (Leshem and Schulkin, 2012).

Finally, the study of the inter- and transgenerational origin of age- or stress-related diseases offers the opportunity to identify predictive or diagnostic biomarkers crucial to develop new interventions according to a tailored clinical approach that claims healthy aging and mental health in today's population and future generations.

\section{AUTHOR CONTRIBUTIONS}

DC and LP coordinated the work, wrote, and edited the article. $\mathrm{EB}, \mathrm{FG}, \mathrm{PC}$, and DL contributed in writing the article.

Belsky, J., Jonassaint, C., Pluess, M., Stanton, M., Brummett, B., and Williams, R. (2009). Vulnerability genes or plasticity genes? Mol. Psychiatry 14, 746-754. doi: $10.1038 / \mathrm{mp} .2009 .44$

Belsky, J., and Pluess, M. (2009). Beyond diathesis stress: differential susceptibility to environmental influences. Psychol. Bull. 135, 885-908. doi: 10.1037/ a0017376

Benito, E., Kerimoglu, C., Ramachandran, B., Pena-Centeno, T., Jain, G., Stilling, R. M., et al. (2018). RNA-dependent intergenerational inheritance of enhanced synaptic plasticity after environmental enrichment. Cell Rep. 23, 546-554. doi: 10.1016/j.celrep.2018.03.059

Bick-Sander, A., Steiner, B., Wolf, S. A., Babu, H., and Kempermann, G. (2006). Running in pregnancy transiently increases postnatal hippocampal neurogenesis in the offspring. Proc. Natl. Acad. Sci. U.S.A. 103, 3852-3857. doi: 10.1073/pnas.0502644103

Bielas, H., Arck, P., Bruenahl, C. A., Walitza, S., and Grünblatt, E. (2014). Prenatal stress increases the striatal and hippocampal expression of correlating c-FOS and serotonin transporters in murine offspring. Int. J. Dev. Neurosci. 38, 30-35. doi: 10.1016/j.ijdevneu.2014.07.006

Boersma, G. J., and Tamashiro, K. L. (2015). Individual differences in the effects of prenatal stress exposure in rodents. Neurobiol. Stress 1, 100-108. doi: 10.1016/j. ynstr.2014.10.006

Bohacek, J., and Mansuy, I. M. (2015). Molecular insights into transgenerational non-genetic inheritance of acquired behaviours. Nat. Rev. Genet. 16, 641-652. doi: $10.1038 / \mathrm{nrg} 3964$

Bosch, O. J. (2013). Maternal aggression in rodents: brain oxytocin and vasopressin mediate pup defence. Philos. Trans. R. Soc. Lond. B Biol. Sci. 368:20130085. doi: 10.1098/rstb.2013.0085

Branchi, I., D’Andrea, I., Cirulli, F., Lipp, H. P., and Alleva, E. (2010). Shaping brain development: mouse communal nesting blunts adult neuroendocrine and behavioral response to social stress and modifies chronic antidepressant treatment outcome. Psychoneuroendocrinology 35, 743-751. doi: 10.1016/j. psyneuen.2009.10.016

Brillaud, E., Morillion, D., and de Seze, R. (2005). Modest environmental enrichment: effect of a radial maze validation and well being of rats. Brain Res. 1054, 174-182. doi: 10.1016/j.brainres.2005.06.069

Brinks, V., van der Mark, M., de Kloet, R., and Oitzl, M. (2007). Emotion and cognition in high and low stress sensitive mouse strains: a combined neuroendocrine and behavioral study in BALB/c and C57BL/6J mice. Front. Behav. Neurosci. 1:8. doi: 10.3389/neuro.08.008.2007 
Brodkin, E. S. (2007). BALB/c mice: low sociability and other phenotypes that may be relevant to autism. Behav. Brain Res. 176, 53-65. doi: 10.1016/j.bbr.2006.06. 025

Byrd, R. S., and Weitzman, M. L. (1994). Predictors of early grade retention among children in the United States. Pediatrics 93, 481-487.

Caldji, C., Hellstrom, I. C., Zhang, T. Y., Diorio, J., and Meaney, M. J. (2011). Environmental regulation of the neural epigenome. FEBS Lett. 585, 2049-2058. doi: 10.1016/j.febslet.2011.03.032

Campos, E. I., Stafford, J. M., and Reinberg, D. (2014). Epigenetic inheritance: histone bookmarks across generations. Trends Cell Biol. 24, 664-674. doi: 10. 1016/j.tcb.2014.08.004

Cancedda, L., Putignano, E., Sale, A., Viegi, A., Berardi, N., and Maffei, L. (2004). Acceleration of visual system development by environmental enrichment. J. Neurosci. 24, 4840-4848. doi: 10.1523/JNEUROSCI.0845-04.2004

Caporali, P., Cutuli, D., Gelfo, F., Laricchiuta, D., Foti, F., De Bartolo, P., et al. (2014). Pre-reproductive maternal enrichment influences offspring developmental trajectories: motor behavior and neurotrophin expression. Front. Behav. Neurosci. 8:195. doi: 10.3389/fnbeh.2014.00195

Caporali, P., Cutuli, D., Gelfo, F., Laricchiuta, D., Foti, F., De Bartolo, P., et al. (2015). Interaction does count: a cross-fostering study on transgenerational effects of pre-reproductive maternal enrichment. Front. Behav. Neurosci. 9:320. doi: 10.3389/fnbeh.2015.00320

Champagne, F. A. (2008). Epigenetic mechanisms and the transgenerational effects of maternal care. Front. Neuroendocrinol. 29, 386-397. doi: 10.1016/j.yfrne. 2008.03.003

Champagne, F. A., and Curley, J. P. (2009). Epigenetic mechanisms mediating the long-term effects of maternal care on development. Neurosci. Biobehav. Rev. 33, 593-600. doi: 10.1016/j.neubiorev.2007.10.009

Champagne, F. A., and Meaney, M. J. (2007). Transgenerational effects of social environment on variations in maternal care and behavioral response to novelty. Behav. Neurosci. 121, 1353-1363. doi: 10.1037/0735-7044.121. 6.1353

Connors, E. J., Migliore, M. M., Pillsbury, S. L., Shaik, A. N., and Kentner, A. C. (2015). Environmental enrichment models a naturalistic form of maternal separation and shapes the anxiety response patterns of offspring. Psychoneuroendocrinology 52, 153-167. doi: 10.1016/j.psyneuen.2014.10.021

Cowansage, K. K., LeDoux, J. E., and Monfils, M. H. (2010). Brain-derived neurotrophic factor: a dynamic gatekeeper of neural plasticity. Curr. Mol. Pharmacol. 3, 12-29. doi: 10.2174/1874467211003010012

Crespi, B. J. (2016). Oxytocin, testosterone, and human social cognition. Biol. Rev. Camb. Philos. Soc. 91, 390-408. doi: 10.1111/brv.12175

Crofton, E. J., Zhang, Y., and Green, T. A. (2015). Inoculation stress hypothesis of environmental enrichment. Neurosci. Biobehav. Rev. 49, 19-31. doi: 10.1016/j. neubiorev.2014.11.017

Curley, J. P., Barton, S., Surani, A., and Keverne, E. B. (2004). Coadaptation in mother and infant regulated by a paternally expressed imprinted gene. Proc. Biol. Sci. 271, 1303-1309. doi: 10.1098/rspb.2004.2725

Curley, J. P., Davidson, S., Bateson, P., and Champagne, F. A. (2009). Social enrichment during postnatal development induces transgenerational effects on emotional and reproductive behavior in mice. Front. Behav. Neurosci. 3:25. doi: 10.3389/neuro.08.025.2009

Cutuli, D., Berretta, E., Caporali, P., Sampedro-Piquero, P., De Bartolo, P., Laricchiuta, D., et al. (2018). Effects of pre-reproductive maternal enrichment on maternal care, offspring's play behavior and oxytocinergic neurons. Neuropharmacology doi: 10.1016/j.neuropharm.2018.02.015 [Epub ahead of print].

Cutuli, D., Berretta, E., Pasqualini, G., De Bartolo, P., Caporali, P., Laricchiuta, D., et al. (2017). Influence of Pre-reproductive maternal enrichment on coping response to stress and expression of c-Fos and glucocorticoid receptors in adolescent offspring. Front. Behav. Neurosci. 11:73. doi: 10.3389/fnbeh.2017. 00073

Cutuli, D., Caporali, P., Gelfo, F., Angelucci, F., Laricchiuta, D., Foti, F., et al. (2015). Pre-reproductive maternal enrichment influences rat maternal care and offspring developmental trajectories: behavioral performances and neuroplasticity correlates. Front. Behav. Neurosci. 9:66. doi: 10.3389/fnbeh. 2015.00066

Cutuli, D., Rossi, S., Burello, L., Laricchiuta, D., De Chiara, V., Foti, F., et al. (2011). Before or after does it matter? Different protocols of environmental enrichment differently influence motor, synaptic and structural deficits of cerebellar origin. Neurobiol. Dis. 42, 9-20. doi: 10.1016/j.nbd.2010.12.007

Cymerblit-Sabba, A., Lasri, T., Gruper, M., Aga-Mizrachi, S., Zubedat, S., and Avital, A. (2013). Prenatal Enriched Environment improves emotional and attentional reactivity to adulthood stress. Behav. Brain Res. 241, 185-190. doi: 10.1016/j.bbr.2012.12.019

Daskalakis, N. P., Bagot, R. C., Parker, K. J., Vinkers, C. H., and de Kloet, E. R. (2013). The three-hit concept of vulnerability and resilience: toward understanding adaptation to early-life adversity outcome. Psychoneuroendocrinology 38, 1858-1873. doi: 10.1016/j.psyneuen.2013. 06.008

Daskalakis, N. P., Oitzl, M. S., Schächinger, H., Champagne, D. L., and Ronald de Kloet, E. (2012). Testing the cumulative stress and mismatch hypotheses of psychopathology in a rat model of early-life adversity. Physiol. Behav. 106, 707-721. doi: 10.1016/j.physbeh.2012.01.015

De Kloet, E. R., Joëls, M., and Holsboer, F. (2005). Stress and the brain: from adaptation to disease. Nat. Rev. Neurosci. 6, 463-475. doi: 10.1038/ nrn1683

De Souza, S. L., Nogueira, M. I., De Jesus Deiró, T. C. B., De Castro, F. M. H. M., Da Silva, C. M., Da Silva, M. C., et al. (2004). Differential effects on somatic and reflex development by chronic clomipramine treatment. Physiol. Behav. 82, 375-379. doi: 10.1016/j.physbeh.2004.04.007

Dell, P. A., and Rose, F. D. (1987). Transfer of effects from environmentally enriched and impoverished female rats to future offspring. Physiol. Behav. 39, 187-190. doi: 10.1016/0031-9384(87)90008-4

Dezsi, G., Ozturk, E., Salzberg, M. R., Morris, M., O’Brien, T. J., and Jones, N. C. (2016). Environmental enrichment imparts disease-modifying and transgenerational effects on genetically-determined epilepsy and anxiety. Neurobiol. Dis. 93, 129-136. doi: 10.1016/j.nbd.2016.05.005

Di Segni, M., Andolina, D., Luchetti, A., Babicola, L., D’Apolito, L. I., Pascucci, T., et al. (2016). Unstable maternal environment affects stress response in adult mice in a genotype-dependent manner. Cereb. Cortex 26, 4370-4380. doi: 10.1093/cercor/bhv204

Dröscher, A. (2015). Of germ-plasm and zymoplasm: august Weismann, Carlo Emery and the debate about the transmission of acquired characteristics. Hist. Philos. Life Sci. 36, 394-403. doi: 10.1007/s40656-014-0046-6

Durán-Carabali, L. E., Arcego, D. M., Odorcyk, F. K., Reichert, L., Cordeiro, J. L., Sanches, E. F., et al. (2018). Prenatal and early postnatal environmental enrichment reduce acute cell death and prevent neurodevelopment and memory impairments in rats submitted to neonatal hypoxia ischemia. Mol. Neurobiol. 55, 3627-3641. doi: 10.1007/s12035-017-0604-5

Enthoven, L., Schmidt, M. V., Cheung, Y. H., van der Mark, M. H., de Kloet, E. R., and Oitzl, M. S. (2010). Ontogeny of the HPA axis of the CD1 mouse following $24 \mathrm{~h}$ maternal deprivation at pnd 3. Int. J. Dev. Neurosci. 28, 217-224. doi: 10.1016/j.ijdevneu.2009.10.006

Essex, M. J., Thomas Boyce, W., Hertzman, C., Lam, L. L., Armstrong, J. M., Neumann, S. M. A., et al. (2013). Epigenetic vestiges of early developmental adversity: childhood stress exposure and DNA methylation in adolescence. Child Dev. 84, 58-75. doi: 10.1111/j.1467-8624.2011.01641.x

Franklin, T. B., and Mansuy, I. M. (2010). Epigenetic inheritance in mammals: evidence for the impact of adverse environmental effects. Neurobiol. Dis. 39, 61-65. doi: 10.1016/j.nbd.2009.11.012

Franklin, T. B., Russig, H., Weiss, I. C., Grff, J., Linder, N., Michalon, A., et al. (2010). Epigenetic transmission of the impact of early stress across generations. Biol. Psychiatry 68, 408-415. doi: 10.1016/j.biopsych.2010. 05.036

Gapp, K., Bohacek, J., Grossmann, J., Brunner, A. M., Manuella, F., Nanni, P., et al. (2016). Potential of environmental enrichment to prevent transgenerational effects of paternal trauma. Neuropsychopharmacology 41, 2749-2758. doi: 10. 1038/npp.2016.87

Gelfo, F., Cutuli, D., Foti, F., Laricchiuta, D., De Bartolo, P., Caltagirone, C., et al. (2011). Enriched environment improves motor function and increases neurotrophins in hemicerebellar lesioned rats. Neurorehabil. Neural Repair 25, 243-252. doi: 10.1177/1545968310380926

Gelfo, F., Mandolesi, L., Serra, L., Sorrentino, G., and Caltagirone, C. (2018). The neuroprotective effects of experience on cognitive functions: evidence from animal studies on the neurobiological bases of brain reserve. Neuroscience 370, 218-235. doi: 10.1016/j.neuroscience.2017.07.065 
Girbovan, C., and Plamondon, H. (2013). Environmental enrichment in female rodents: considerations in the effects on behavior and biochemical markers. Behav. Brain Res. 253, 178-190. doi: 10.1016/j.bbr.2013. 07.018

Glover, V. (2011). Annual research review: prenatal stress and the origins of psychopathology: an evolutionary perspective. J. Child Psychol. Psychiatry 52, 356-367. doi: 10.1111/j.1469-7610.2011.02371.x

Gomes Da Silva, S., De Almeida, A. A., Fernandes, J., Lopim, G. M., Cabral, F. R., Scerni, D. A., et al. (2016). Maternal exercise during pregnancy increases BDNF levels and cell numbers in the hippocampal formation but not in the cerebral cortex of adult rat offspring. PLoS One 11:e0147200. doi: 10.1371/journal.pone. 0147200

Grégoire, C. A., Bonenfant, D., Le Nguyen, A., Aumont, A., and Fernandes, K. J. (2014). Untangling the influences of voluntary running, environmental complexity, social housing and stress on adult hippocampal neurogenesis. PLoS One 9:e86237. doi: 10.1371/journal.pone.0086237

Guzzetta, A., Baldini, S., Bancale, A., Baroncelli, L., Ciucci, F., Ghirri, P., et al. (2009). Massage accelerates brain development and the maturation of visual function. J. Neurosci. 29, 6042-6051. doi: 10.1523/JNEUROSCI.5548-08.2009

Hathaway, G. A., Faykoo-Martinez, M., Peragine, D. E., Mooney, S. J., and Holmes, M. M. (2016). Subcaste differences in neural activation suggest a prosocial role for oxytocin in eusocial naked mole-rats. Horm. Behav. 79, 1-7. doi: 10.1016/j. yhbeh.2015.12.001

Heiderstadt, K. M., Vandenbergh, D. J., Gyekis, J. P., and Blizard, D. A. (2014). Communal nesting increases pup growth but has limited effects on adult behavior and neurophysiology in inbred mice. J. Am. Assoc. Lab. Anim. Sci. 53, $152-160$.

Herman, J. P., McKlveen, J. M., Ghosal, S., Kopp, B., Wulsin, A., Makinson, R., et al. (2016). Regulation of the hypothalamic-pituitary-adrenocortical stress response. Compr. Physiol. 6, 603-621. doi: 10.1002/cphy.c150015

Herring, A., Donath, A., Yarmolenko, M., Uslar, E., Conzen, C., Kanakis, D., et al. (2012). Exercise during pregnancy mitigates Alzheimer-like pathology in mouse offspring. FASEB J. 26, 117-128. doi: 10.1096/fj.11-193193

Huzard, D., Mumby, D. G., Sandi, C., Poirier, G. L., and van der Kooij, M. A. (2015). The effects of extrinsic stress on somatic markers and behavior are dependent on animal housing conditions. Physiol. Behav. 151, 238-245. doi: 10.1016/j.physbeh.2015.07.018

Ito, A., Kikusui, T., Takeuchi, Y., and Mori, Y. (2006). Effects of early weaning on anxiety and autonomic responses to stress in rats. Behav. Brain Res. 171, 87-93. doi: 10.1016/j.bbr.2006.03.023

Jirtle, R. L., and Skinner, M. K. (2007). Environmental epigenomics and disease susceptibility. Nat. Rev. Genet. 8, 253-262. doi: 10.1038/nrg2045

Karatsoreos, I. N., and McEwen, B. S. (2011). Psychobiological allostasis: resistance, resilience and vulnerability. Trends Cogn. Sci. 15, 576-584. doi: 10.1016/j.tics. 2011.10.005

Kempermann, G., Kuhn, H. G., and Gage, F. H. (1997). More hippocampal neurons in adult mice living in an enriched environment. Nature 386, 493-495. doi: $10.1038 / 386493 \mathrm{a} 0$

Kikusui, T., Nakamura, K., Kakuma, Y., and Mori, Y. (2006). Early weaning augments neuroendocrine stress responses in mice. Behav. Brain Res. 175, 96-103. doi: 10.1016/j.bbr.2006.08.007

Kiss, P., Vadasz, G., Kiss-Illes, B., Horvath, G., Tamas, A., Reglodi, D., et al. (2013). Environmental enrichment decreases asphyxia-induced neurobehavioral developmental delay in neonatal rats. Int. J. Mol. Sci. 14, 22258-22273. doi: 10.3390/ijms141122258

Kiyono, S., Seo, M. L., Shibagaki, M., and Inouye, M. (1985). Facilitative effects of maternal environmental enrichment on maze learning in rat offspring. Physiol. Behav. 34, 431-435. doi: 10.1016/0031-9384(85)90207-0

Kobilo, T., Liu, Q. R., Gandhi, K., Mughal, M., Shaham, Y., and van Praag, H. (2011). Running is the neurogenic and neurotrophic stimulus in environmental enrichment. Learn. Mem. 18, 605-609. doi: 10.1101/lm.2283011

Kollack-Walker, S., Watson, S. J., and Akil, H. (1997). Social stress in hamsters: defeat activates specific neurocircuits within the brain. J. Neurosci. 17, 88428855. doi: 10.1016/0031-9384(87)90137-5

Koo, J. W., Park, C. H., Choi, S. H., Kim, N. J., Kim, H. S., Choe, J. C., et al. (2003). The postnatal environment can counteract prenatal effects on cognitive ability, cell proliferation, and synaptic protein expression. FASEB J. 17, 1556-1558. doi: 10.1096/fj.02-1032fje
Kristal, M. B. (2009). The biopsychology of maternal behavior in nonhuman mammals. ILAR J. 50, 51-63. doi: 10.1093/ilar.50.1.51

Kundakovic, M., and Champagne, F. A. (2015). Early-life experience, Epigenetics, and the developing brain. Neuropsychopharmacology 40, 141-153. doi: 10.1038/ npp. 2014.140

Kundakovic, M., Lim, S., Gudsnuk, K., and Champagne, F. A. (2013). Sex-specific and strain-dependent effects of early life adversity on behavioral and epigenetic outcomes. Front. Psychiatry 4:78. doi: 10.3389/fpsyt.2013.00078

Larsson, F., Winblad, B., and Mohammed, A. H. (2002). Psychological stress and environmental adaptation in enriched vs. Impoverished housed rats. Pharmacol. Biochem. Behav. 73, 193-207. doi: 10.1016/S0091-3057(02)00 782-7

Laviola, G., Rea, M., Morley-Fletcher, S., Di Carlo, S., Bacosi, A., De Simone, R., et al. (2004). Beneficial effects of enriched environment on adolescent rats from stressed pregnancies. Eur. J. Neurosci. 20, 1655-1664. doi: 10.1111/j.1460-9568. 2004.03597.x

Lee, H. H., Kim, H., Lee, J. W., Kim, Y. S., Yang, H. Y., Chang, H. K., et al. (2006). Maternal swimming during pregnancy enhances short-term memory and neurogenesis in the hippocampus of rat pups. Brain Dev. 28, 147-154. doi: 10.1016/j.braindev.2005.05.007

Lee, H. J., Macbeth, A. H., Pagani, J. H., and Scott Young, W. (2009). Oxytocin: the great facilitator of life. Prog. Neurobiol. 88, 127-151. doi: 10.1016/j.pneurobio. 2009.04.001

Lehrner, A., Bierer, L. M., Passarelli, V., Pratchett, L. C., Flory, J. D., Bader, H. N., et al. (2014). Maternal PTSD associates with greater glucocorticoid sensitivity in offspring of Holocaust survivors. Psychoneuroendocrinology 40, 213-220. doi: 10.1016/j.psyneuen.2013.11.019

Leshem, M., and Schulkin, J. (2012). Transgenerational effects of infantile adversity and enrichment in male and female rats. Dev. Psychobiol. 54, 169-186. doi: 10.1002/dev.20592

Lim, J. P., and Brunet, A. (2013). Bridging the transgenerational gap with epigenetic memory. Trends Genet. 29, 176-186. doi: 10.1016/j.tig.2012.12.008

Lim, M. M., and Young, L. J. (2006). Neuropeptidergic regulation of affiliative behavior and social bonding in animals. Horm. Behav. 50, 506-517. doi: 10 . 1016/j.yhbeh.2006.06.028

Livingston-Thomas, J., Nelson, P., Karthikeyan, S., Antonescu, S., Jeffers, M. S., and Marzolini, S. (2016). Exercise and environmental enrichment as enablers of task-specific neuroplasticity and stroke recovery. Neurotherapeutics 13, 395402. doi: 10.1007/s13311-016-0423-9

Lo, C. L., and Zhou, F. C. (2014). Environmental alterations of epigenetics prior to the birth. Int. Rev. Neurobiol. 115, 1-49. doi: 10.1016/B978-0-12-801311-3. 00001-9

Mandolesi, L., Gelfo, F., Serra, L., Montuori, S., Polverino, A., Curcio, G., et al. (2017). Environmental factors promoting neural plasticity: insights from animal and human studies. Neural Plast. 2017:7219461. doi: 10.1155/2017/ 7219461

Marcon, M., Mocelin, R., Benvenutti, R., Costa, T., Herrmann, A. P., and de Oliveira, D. L. (2018). Environmental enrichment modulates the response to chronic stress in zebrafish. J. Exp. Biol. 221:jeb176735. doi: 10.1242/jeb.176735

Marshall, D. J., and Uller, T. (2007). When is a maternal effect adaptive? Oikos 116, 1957-1963. doi: 10.1111/j.2007.0030-1299.16203.x

Maruoka, T., Kodomari, I., Yamauchi, R., Wada, E., and Wada, K. (2009). Maternal enrichment affects prenatal hippocampal proliferation and open-field behaviors in female offspring mice. Neurosci. Lett. 454, 28-32. doi: 10.1016/j.neulet.2009. 02.052

Mashoodh, R., Franks, B., Curley, J. P., and Champagne, F. A. (2012). Paternal social enrichment effects on maternal behavior and offspring growth. Proc. Natl. Acad. Sci. U.S.A. 109, 17232-17238. doi: 10.1073/pnas. 1121083109

McCreary, J. K., and Metz, G. A. S. (2016). Environmental enrichment as an intervention for adverse health outcomes of prenatal stress. Environ. Epigenet. 2:dvw013. doi: 10.1093/eep/dvw013

McKim, M., and Thompson, W. R. (1975). Prenatal maternal enrichment and restriction in rats: effects on biological and foster offspring. Bull. Psychon. Soc. 5, 259-260. doi: 10.3758/BF03337626

Meaney, M. J. (2001). Maternal care, gene expression, and the transmission of individual differences in stress reactivity across generations. Ann. Rev. Neurosci. 24, 1161-1192. doi: 10.1146/annurev.neuro.24.1.1161 
Meaney, M. J. (2010). Epigenetics and the biological definition of gene $\mathrm{X}$ environment interactions. Child Dev. 81, 41-79. doi: 10.1111/j.1467-8624.2009. 01381.x

Mifsud, K. R., and Reul, J. M. H. M. (2018). Mineralocorticoid and glucocorticoid receptor-mediated control of genomic responses to stress in the brain. Stress 4, 1-14. doi: 10.1080/10253890.2018.1456526

Mo, C., Renoir, T., and Hannan, A. J. (2016). What's wrong with my mouse cage? Methodological considerations for modeling lifestyle factors and geneenvironment interactions in mice. J. Neurosci. Methods 265, 99-108. doi: 10. 1016/j.jneumeth.2015.08.008

Moncek, F., Duncko, R., Johansson, B. B., and Jezova, D. (2004). Effect of environmental enrichment on stress related systems in rats. J. Neuroendocrinol. 16, 423-431. doi: 10.1111/j.1365-2826.2004.01173.x

Mooney, S. M., and Varlinskaya, E. I. (2011). Acute prenatal exposure to ethanol and social behavior: effects of age, sex, and timing of exposure. Behav. Brain Res. 216, 358-364. doi: 10.1016/j.bbr.2010.08.014

Morley-Fletcher, S., Rea, M., Maccari, S., and Laviola, G. (2003). Environmental enrichment during adolescence reverses the effects of prenatal stress on play behaviour and HPA axis reactivity in rats. Eur. J. Neurosci. 18, 3367-3374. doi: 10.1111/j.1460-9568.2003.03070.x

Mosaferi, B., Babri, S., Mohaddes, G., Khamnei, S., and Mesgari, M. (2015). Postweaning environmental enrichment improves BDNF response of adult male rats. Int. J. Dev. Neurosci. 46, 108-114. doi: 10.1016/j.ijdevneu.2015.07.008

Mulligan, C. J., D’Errico, N. C., Stees, J., and Hughes, D. A. (2012). Methylation changes at $\mathrm{NR} 3 \mathrm{C} 1$ in newborns associate with maternal prenatal stress exposure and newborn birth weight. Epigenetics 7, 853-857. doi: 10.4161/epi.21180

Mustroph, M. L., Chen, S., Desai, S. C., Cay, E. B., DeYoung, E. K., and Rhodes, J. S. (2012). Aerobic exercise is the critical variable in an enriched environment that increases hippocampal neurogenesis and water maze learning in male C57BL/6J mice. Neuroscience 219, 62-71. doi: 10.1016/j.neuroscience.2012.06.007

Mychasiuk, R., Zahir, S., Schmold, N., Ilnytskyy, S., Kovalchuk, O., and Gibb, R. (2012). Parental enrichment and offspring development: modifications to brain, behavior and the epigenome. Behav. Brain Res. 228, 294-298. doi: 10.1016/j.bbr. 2011.11.036

Nederhof, E., and Schmidt, M. V. (2012). Mismatch or cumulative stress: toward an integrated hypothesis of programming effects. Physiol. Behav. 106, 691-700. doi: 10.1016/j.physbeh.2011.12.008

Nithianantharajah, J., and Hannan, A. J. (2006). Enriched environments, experience-dependent plasticity and disorders of the nervous system. Nat. Rev. Neurosci. 7, 697-709. doi: 10.1038/nrn1970

Nithianantharajah, J., and Hannan, A. J. (2009). The neurobiology of brain and cognitive reserve: mental and physical activity as modulators of brain disorders. Prog. Neurobiol. 89, 369-382. doi: 10.1016/j.pneurobio.2009.10.001

Novkovic, T., Mittmann, T., and Manahan-Vaughan, D. (2015). BDNF contributes to the facilitation of hippocampal synaptic plasticity and learning enabled by environmental enrichment. Hippocampus 25, 1-15. doi: 10.1002/hipo.22342

Olson, A. K., Eadie, B. D., Ernst, C., and Christie, B. R. (2006). Environmental enrichment and voluntary exercise massively increase neurogenesis in the adult hippocampus via dissociable pathways. Hippocampus 16, 250-260. doi: 10.1002/ hipo. 20157

Olsson, A. S., and Dahlborn, K. (2002). Improving housing conditions for laboratory mice: a review of 'environmental enrichment'. Lab. Anim. 36, 243270. doi: 10.1258/002367702320162379

Park, H., and Poo, M. M. (2013). Neurotrophin regulation of neural circuit development and function. Nat. Rev. Neurosci. 14, 7-23. doi: 10.1038/nrn3379

Park, J. W., Kim, M. H., Eo, S. J., Lee, E. H., Kang, J. S., Chang, H. K., et al. (2013). Maternal exercise during pregnancy affects mitochondrial enzymatic activity and biogenesis in offspring brain. Int. J. Neurosci. 123, 253-264. doi: 10.3109/00207454.2012.755969

Parnpiansil, P., Jutapakdeegul, N., Chentanez, T., and Kotchabhakdi, N. (2003). Exercise during pregnancy increases hippocampal brain-derived neurotrophic factor mRNA expression and spatial learning in neonatal rat pup. Neurosci. Lett. 352, 45-48. doi: 10.1016/j.neulet.2003.08.023

Peña, Y., Prunell, M., Rotllant, D., Armario, A., and Escorihuela, R. M. (2009). Enduring effects of environmental enrichment from weaning to adulthood on pituitary-adrenal function, pre-pulse inhibition and learning in male and female rats. Psychoneuroendocrinology 34, 1390-1404. doi: 10.1016/j.psyneuen.2009. 04.019
Petrosini, L., De Bartolo, P., Foti, F., Gelfo, F., Cutuli, D., Leggio, M. G., et al. (2009). On whether the environmental enrichment may provide cognitive and brain reserves. Brain Res. Rev. 61, 221-239. doi: 10.1016/j.brainresrev.2009.07.002

Pham, T. M., Söderström, S., Winblad, B., and Mohammed, A. H. (1999). Effects of environmental enrichment on cognitive function and hippocampal NGF in the non-handled rats. Behav. Brain Res. 103, 63-70. doi: 10.1016/S0166-4328(99) 00019-4

Picciolini, O., Porro, M., Meazza, A., Giannì, M. L., Rivoli, C., Lucco, G., et al. (2014). Early exposure to maternal voice: effects on preterm infants development. Early Hum. Dev. 90, 287-292. doi: 10.1016/j.earlhumdev.2014. 03.003

Plagge, A., Gordon, E., Dean, W., Boiani, R., Cinti, S., Peters, J., et al. (2004). The imprinted signaling protein XL alpha s is required for postnatal adaptation to feeding. Nat. Genet. 36, 818-826. doi: 10.1038/ng1397

Prathanee, B., Purdy, S. C., Thinkhamrop, B., Chaimay, B., Ruangdaraganon, N., Mosuwan, L., et al. (2009). Early language delay and predictive factors in children aged 2 years. J. Med. Assoc. Thai. 92, 930-938.

Prizak, R., Ezard, T. H. G., and Hoyle, R. B. (2014). Fitness consequences of maternal and grandmaternal effects. Ecol. Evol. 4, 3139-3145. doi: 10.1002/ece3. 1150

Rosenfeld, A., and Weller, A. (2012). Behavioral effects of environmental enrichment during gestation in WKY and Wistar rats. Behav. Brain Res. 233, 245-255. doi: 10.1016/j.bbr.2012.05.006

Rosenzweig, M. R., Bennett, E. L., and Krech, D. (1964). Cerebral effects of environmental complexity and training among adult rats. J. Comp. Physiol. Psychol. 57, 438-439. doi: 10.1037/h0046387

Ruan, Z. L., Liu, L., Strodl, E., Fan, L. J., Yin, X. N., Wen, G. M., et al. (2018). Antenatal training with music and maternal talk concurrently may reduce autistic-like behaviors at around 3 years of age. Front. Psychiatry 8:305. doi: $10.3389 /$ fpsyt.2017.00305

Sale, A. (2018). A systematic look at environmental modulation and its impact in brain development. Trends Neurosci. 41, 4-17. doi: 10.1016/j.tins.2017.10.004

Sale, A., Berardi, N., and Maffei, L. (2014). Environment and brain plasticity: towards an endogenous pharmacotherapy. Physiol. Rev. 94, 189-234. doi: 10.1152/physrev.00036.2012

Sale, A., Putignano, E., Cancedda, L., Landi, S., Cirulli, F., Berardi, N., et al. (2004). Enriched environment and acceleration of visual system development. Neuropharmacology 47, 649-660. doi: 10.1016/j.neuropharm.2004.07.008

Sampedro-Piquero, P., and Begega, A. (2017). Environmental enrichment as a positive behavioral intervention across the lifespan. Curr. Neuropharmacol. 15, 459-470. doi: 10.2174/1570159X14666160325115909

Sanchez, M. M. (2006). The impact of early adverse care on HPA axis development: nonhuman primate models. Horm. Behav. 50, 623-631. doi: 10.1016/j.yhbeh. 2006.06.012

Sapolsky, R. M. (2015). Stress and the brain: individual variability and the invertedU. Nat. Neurosci. 18, 1344-1346. doi: 10.1038/nn.4109

Schilling, G., Savonenko, A. V., Coonfield, M. L., Morton, J. L., Vorovich, E., Gale, A., et al. (2004). Environmental, pharmacological, and genetic modulation of the HD phenotype in transgenic mice. Exp. Neurol. 187, 137-149. doi: 10.1016/j.expneurol.2004.01.003

Schuch, C. P., Diaz, R., Deckmann, I., Rojas, J. J., Deniz, B. F., and Pereira, L. O. (2016). Early environmental enrichment affects neurobehavioral development and prevents brain damage in rats submitted to neonatal hypoxia-ischemia. Neurosci. Lett. 617, 101-107. doi: 10.1016/j.neulet.2016.02.015

Shachar-Dadon, A., Schulkin, J., and Leshem, M. (2009). Adversity before conception will affect adult progeny in rats. Dev. Psychol. 45, 9-16. doi: 10.1037/ a0014030

Short, A. K., Yeshurun, S., Powell, R., Perreau, V. M., Fox, A., Kim, J. H., et al. (2017). Exercise alters mouse sperm small noncoding RNAs and induces a transgenerational modification of male offspring conditioned fear and anxiety. Transl. Psychiatry 7:e1114. doi: 10.1038/tp.2017.82

Simpson, J., and Kelly, J. P. (2011). The impact of environmental enrichment in laboratory rats-Behavioural and neurochemical aspects. Behav. Brain Res. 222, 246-264. doi: 10.1016/j.bbr.2011.04.002

Sparling, J. E., Mahoney, M., Baker, S., and Bielajew, C. (2010). The effects of gestational and postpartum environmental enrichment on the mother rat: a preliminary investigation. Behav. Brain Res. 208, 213-223. doi: 10.1016/j.bbr. 2009.11.041 
Swaney, W. T. (2011). Genomic imprinting and mammalian reproduction. Horm. Behav. 59, 369-374. doi: 10.1016/j.yhbeh.2010.05.012

Swartz, J. R., Knodt, A. R., Radtke, S. R., and Hariri, A. R. (2018). Postsecondary maternal education buffers against neural risk for psychological vulnerability to future life stress. Neuropsychologia 109, 134-139. doi: 10.1016/ j.neuropsychologia.2017.12.019

Sztainberg, Y., Kuperman, Y., Tsoory, M., Lebow, M., and Chen, A. (2010). The anxiolytic effect of environmental enrichment is mediated via amygdalar CRF receptor type 1. Mol. Psychiatry 15, 905-917. doi: 10.1038/mp.2009.151

Taouk, L., and Schulkin, J. (2016). Transgenerational transmission of pregestational and prenatal experience: maternal adversity, enrichment, and underlying epigenetic and environmental mechanisms. J. Dev. Orig. Health Dis. 7, 588-601. doi: 10.1017/S2040174416000416

Thayer, Z. M., and Kuzawa, C. W. (2011). Biological memories of past environments: epigenetic pathways to health disparities. Epigenetics 6, 798-803. doi: $10.4161 /$ epi.6.7.16222

Todeschin, A. S., Winkelmann-Duarte, E. C., Jacob, M. H. V., Aranda, B. C. C., Jacobs, S., Fernandes, M. C., et al. (2009). Effects of neonatal handling on social memory, social interaction, and number of oxytocin and vasopressin neurons in rats. Horm. Behav. 56, 93-100. doi: 10.1016/j.yhbeh.2009.03.006

Tollenaar, M. S., Beijers, R., Jansen, J., Riksen-Walraven, J. M. A., and De Weerth, C. (2011). Maternal prenatal stress and cortisol reactivity to stressors in human infants. Stress 14, 53-65. doi: 10.3109/10253890.2010.499485

Trezza, V., Campolongo, P., and Vanderschuren, L. J. M. J. (2011). Evaluating the rewarding nature of social interactions in laboratory animals. Dev. Cogn. Neurosci. 1, 444-458. doi: 10.1016/j.dcn.2011.05.007

van Praag, H., Kempermann, G., and Gage, F. H. (1999). Running increases cell proliferation and neurogenesis in the adult mouse dentate gyrus. Nat. Neurosci. 2, 266-270. doi: 10.1038/6368

Vanderschuren, L. J., Niesink, R. J., and Van Ree, J. M. (1997). The neurobiology of social play behavior in rats. Neurosci. Biobehav. Rev. 21, 309-326. doi: 10.1016/ S0149-7634(96)00020-6

Vanderschuren, L. J. M. J., Achterberg, E. J. M., and Trezza, V. (2016). The neurobiology of social play and its rewarding value in rats. Neurosci. Biobehav. Rev. 70, 86-105. doi: 10.1016/j.neubiorev.2016.07.025

Veenema, A. H. (2009). Early life stress, the development of aggression and neuroendocrine and neurobiological correlates: what can we learn from animal models? Front. Neuroendocrinol. 30:497-518. doi: 10.1016/j.yfrne.2009.03.003

Veenendaal, M. V., Costello, P. M., Lillycrop, K. A., de Rooij, S. R., van der Post, J. A., Bossuyt, P. M., et al. (2012). Prenatal famine exposure, health in later life and promoter methylation of four candidate genes. J. Dev. Orig. Health Dis. 3 , 450-457. doi: 10.1017/S2040174412000396
Wang, Y., Liu, H., and Sun, Z. (2017). Lamarck rises from his grave: parental environment-induced epigenetic inheritance in model organisms and humans. Biol. Rev. 92, 2084-2111. doi: 10.1111/brv.12322

Weaver, I. C. G., Korgan, A. C., Lee, K., Wheeler, R. V., Hundert, A. S., and Goguen, D. (2017). Stress and the emerging roles of chromatin remodeling in signal integration and stable transmission of reversible phenotypes. Front. Behav. Neurosci. 11:41. doi: 10.3389/fnbeh.2017.00041

Webb, A. R., Heller, H. T., Benson, C. B., and Lahav, A. (2015). Mother's voice and heartbeat sounds elicit auditory plasticity in the human brain before full gestation. Proc. Natl. Acad. Sci. U.S.A. 112, 3152-3157. doi: 10.1073/pnas. 1414924112

Welberg, L., Thrivikraman, K. V., and Plotsky, P. M. (2006). Combined pre- and postnatal environmental enrichment programs the HPA axis differentially in male and female rats. Psychoneuroendocrinology 31, 553-564. doi: 10.1016/j. psyneuen.2005.11.011

Wood, N. I., Glynn, D., and Morton, A. J. (2011). "Brain training" improves cognitive performance and survival in a transgenic mouse model of Huntington's disease. Neurobiol. Dis. 42, 427-437. doi: 10.1016/j.nbd.2011.02. 005

$\mathrm{Xu}, \mathrm{H} ., \mathrm{Li}, \mathrm{L} ., \mathrm{Zhang}, \mathrm{Z}$., and Liu, J. (2016). Is natural experiment a cure? Reexamining the long-term health effects of China's 1959-1961 famine. Soc. Sci. Med. 148, 110-122. doi: 10.1016/j.socscimed.2015.11.028

Yeshurun, S., Short, A. K., Bredy, T. W., Pang, T. Y., and Hannan, A. J. (2017). Paternal environmental enrichment transgenerationally alters affective behavioral and neuroendocrine phenotypes. Psychoneuroendocrinology 77, 225-235. doi: 10.1016/j.psyneuen.2016.11.013

Zuena, A. R., Zinni, M., Giuli, C., Cinque, C., Alemà, G. S., Giuliani, A., et al. (2016). Maternal exposure to environmental enrichment before and during gestation influences behaviour of rat offspring in a sex-specific manner. Physiol. Behav. 163, 274-287. doi: 10.1016/j.physbeh.2016.05.010

Conflict of Interest Statement: The authors declare that the research was conducted in the absence of any commercial or financial relationships that could be construed as a potential conflict of interest.

Copyright (c) 2018 Cutuli, Berretta, Laricchiuta, Caporali, Gelfo and Petrosini. This is an open-access article distributed under the terms of the Creative Commons Attribution License (CC BY). The use, distribution or reproduction in other forums is permitted, provided the original author(s) and the copyright owner(s) are credited and that the original publication in this journal is cited, in accordance with accepted academic practice. No use, distribution or reproduction is permitted which does not comply with these terms. 